\title{
Benefits and harms of the human papillomavirus (HPV) vaccines: systematic review with meta-analyses of trial data from clinical study reports
}

\author{
Lars Jørgensen ${ }^{1} \mathbb{D}$, Peter C. Gøtzsche ${ }^{2,3}$ and Tom Jefferson ${ }^{1 *}$
}

\begin{abstract}
Objective: To assess the benefits and harms of the human papillomavirus (HPV) vaccines.

Data sources: Clinical study reports obtained from the European Medicines Agency and GlaxoSmithKline from 2014 to 2017.

Eligibility criteria: Randomised trials that compared an HPV vaccine with a placebo or active comparator in healthy participants of all ages.

Appraisal and synthesis: Two researchers extracted data and judged risk of bias with the Cochrane tool (version 2011). Risk ratio (RR) estimates were pooled using random-effects meta-analysis.

Outcomes: Clinically relevant outcomes in intention to treat populations-including HPV-related cancer precursors irrespective of involved HPV types, treatment procedures and serious and general harms.

Results: Twenty-four of 50 eligible clinical study reports were obtained with 58,412 pages of 22 trials and 2 followup studies including 95,670 participants: 79,102 females and 16,568 males age 8-72; 393,194 person-years; and 49 months mean weighted follow-up. We judged all 24 studies to be at high risk of bias. Serious harms were incompletely reported for $72 \%$ of participants $(68,610 / 95,670)$. Nearly all control participants received active comparators $(48,289 / 48,595,99 \%)$. No clinical study report included complete case report forms. At 4 years followup, the HPV vaccines reduced HPV-related carcinoma in situ (367 in the HPV vaccine group vs. 490 in the comparator group, RR 0.73 [95\% confidence interval, $\mathrm{Cl}, 0.53$ to 1.00], number needed to vaccinate [NNV] 387, $P=0.05, P^{2}=67 \%$ ) and HPV-related treatment procedures (1018 vs. 1416, RR 0.71 [95\% Cl 0.63 to 0.80], NNV 75, $P<0.00001, P^{2}=45 \%$ ). The HPV vaccines increased serious nervous system disorders (exploratory analysis: 72 vs. 46 , RR 1.49 [1.02 to 2.16], number needed to harm [NNH] 1325, $\left.P=0.040, P^{2}=0 \%\right)$ and general harms $(13,248 \mathrm{vs} .12,394$, RR 1.07 [95\% Cl 1.03 to 1.11], NNH 51, $P=0.0002, P^{2}=77 \%$ ) but did not significantly increase fatal harms (45 vs. 38 , RR 1.19 [95\% Cl 0.65 to 2.19], $P=0.58, P^{2}=30 \%$ ) or serious harms (1404 vs. 1357, RR 1.01 [95\% Cl 0.94 to 1.08], $P=$ $\left.0.79, P^{2}=0 \%\right)$.

\footnotetext{
*Correspondence: tj@cochrane.dk

${ }^{1}$ Nordic Cochrane Centre, Rigshospitalet 7811, Tagensvej 22, 2200

Copenhagen, Denmark

Full list of author information is available at the end of the article
}

(c) The Author(s). 2020 Open Access This article is distributed under the terms of the Creative Commons Attribution 4.0 International License (http://creativecommons.org/licenses/by/4.0/), which permits unrestricted use, distribution, and reproduction in any medium, provided you give appropriate credit to the original author(s) and the source, provide a link to the Creative Commons license, and indicate if changes were made. The Creative Commons Public Domain Dedication waiver (http://creativecommons.org/publicdomain/zero/1.0/) applies to the data made available in this article, unless otherwise stated. 


\begin{abstract}
(Continued from previous page)
Conclusion: At 4 years follow-up, the HPV vaccines decreased HPV-related cancer precursors and treatment procedures but increased serious nervous system disorders (exploratory analysis) and general harms. As the included trials were primarily designed to assess benefits and were not adequately designed to assess harms, the extent to which the HPV vaccines' benefits outweigh their harms is unclear. Limited access to clinical study reports and trial data with case report forms prevented a thorough assessment.

Systematic review registration: CRD42017056093. Our systematic review protocol was registered on PROSPERO in January 2017: https://www.crd.york.ac.uk/PROSPEROFILES/56093_PROTOCOL_20170030.pdf. Two protocol amendments were registered on PROSPERO on November 2017: https://www.crd.york.ac.uk/PROSPEROFILES/56093_PROTOCOL_ 20171116.pdf. Our index of the HPV vaccine studies was published in Systematic Reviews in January 2018: https://doi. org/10.1186/s13643-018-0675-z. A description of the challenges obtaining the data was published in September 2018: https://doi.org/10.1136/bmj.k3694.
\end{abstract}

Keywords: Human papillomavirus vaccine, Systematic review, Meta-analysis, Randomised clinical trial and Clinical study report

\section{Introduction}

The approved human papillomavirus (HPV) vaccinesGlaxoSmithKline's Cervarix ${ }^{\mathrm{Tm}}$ and Merck Sharp and Dohme's Gardasil ${ }^{\mathrm{Tm}}$ and Gardasil $9^{\mathrm{Tm}}$-are considered safe and effective [1-3]. Recent evidence suggests that the vaccines have significant and long-lasting effects ( $>12$ years) on cervical cancer $[4,5]$, better effectiveness when vaccinated below the age of 17 [6], and are possibly able to substantially reduce the global incidence of cervical cancer [7]. However, there are important uncertainties regarding both the benefits and harms of the vaccines.

\section{Uncertainties of the benefits of the HPV vaccines}

The HPV vaccines' regulatory approvals were mainly based on per-protocol populations and surrogate outcomes of HPV-related lesions, e.g. 'cervical intraepithelial neoplasia or worse' $\left(\mathrm{CIN}^{+}\right)$infected with an HPV vaccine-specific HPV type, such as HPV types 16 and 18 that are associated with the majority of HPV-related cancers [8-10]. It was considered unfeasible and unethical to use HPV-related cancer as the primary outcome [11, 12], since it takes many years for cancer to develop after an HPV infection and also because cervical screening is an established secondary prevention method that leads to removal of precancerous lesions before they become cancerous. Up to $15 \%$ of HPV-related cervical cancers may not contain HPV [13], but HPV may be identified in more cases with newer and more sensitive analysis methods [14]. HPV-related lesions are often infected with more than one HPV type, some of which may not be targeted by the vaccines [15]. This makes it impossible to assess which HPV type caused the lesion. The regulatory vaccine approvals were not based on HPV-related lesions irrespective of HPV type in intention to treat populations, and factors such as antigenic changes and herd immunity may be important in the long-term perspective, as the approved HPV vaccines only target up to 9 of the $25 \mathrm{HPV}$ types considered oncogenic [1].

\section{Uncertainties of the harms of the HPV vaccines}

A Cochrane review from 2018 [3] and most large epidemiological studies [16-20] did not find serious or general harms associated with the HPV vaccines. The Cochrane review was mainly based on journal publications that often are influenced by reporting bias [21-24], and epidemiological studies are influenced by confounding [25].

Acknowledged rare serious harms include anaphylaxis and syncope [8-10]. Some case studies have reported rare neurological harms such as postural orthostatic tachycardia syndrome (POTS) $[26,27]$ and complex regional pain syndrome (CRPS) [28]. Cluster analyses of individual case safety reports from the World Health Organisation's (WHOs) VigiBase ${ }^{\circ}$ revealed additional harms-often serious in nature-that overlapped with the symptomatology of POTS and CRPS [29]. Although the European Medicines Agency's (EMA) investigation of POTS and CRPS did not find an association with the HPV vaccines [2], EMA's investigation was based on the HPV vaccine manufacturers' own assessments [30], and about 30 cases of POTS and CRPS were not recognised in the HPV vaccine manufacturers' trials [31, 32]. Other reported rare harms have included chronic fatigue syndrome (CFS), Guillain-Barré syndrome (GBS) and premature ovarian failure (POF) [33-35].

\section{Addressing the uncertainties of the HPV vaccines}

To address the uncertainties of the benefits and harms of the HPV vaccines, we conducted a systematic review 
with meta-analyses of trial data from clinical study reports. As of July 2017, about one third of the HPV vaccine studies had not been published and study results were not posted for about half of the completed studies on ClinicalTrials.gov [36]. Therefore, we based our review on study programmes in order to identify all trials [36] and on clinical study reports [37], as these reports provide vastly more information about a study than a corresponding journal publication [21-24].

\section{Methods}

\section{Search strategy and study eligibility}

Using a six-step process, we constructed and published an index of the HPV vaccine study programmes [36] that included 206 comparative prospective studies (see Fig. 1). Two researchers (LJ and TJ) conducted the six steps that included searches of trial registers, journal publication databases and correspondence with regulators and HPV vaccine manufacturers. It was not feasible to account for duplicate entries, as we indexed studies and searched databases that used different IDs for a unique study (e.g. register ID, study programme ID, manufacturer ID and publication ID) [36].

In May 2014, we requested the study programmes' corresponding clinical study reports from the European Medicines Agency (EMA; via its policy 0043) and obtained those reports that were freely available on GlaxoSmithKline's online trial register. We did not request clinical study reports from the manufacturers, as this would limit our ability to use and share the data [38]. In January 2017, we registered our systematic review protocol in PROSPERO (International prospective register of systematic reviews): CRD42017056093 [37].

We included those trials and their follow-up studies of the 206 comparative studies from our index that were randomised clinical phase II, III or IV trials. We aimed to include studies for which we obtained industry clinical study reports or similar non-industry reports. In the event of no clinical study report being available (for an otherwise eligible trial), we did not include data from the trial publication. We also aimed to include periodical safety update reports. PICO criteria (participants, interventions, comparisons and outcomes) were used to select trials that compared an HPV vaccine with a placebo (normal saline) or active comparator (adjuvant or non-HPV vaccine such as a hepatitis vaccine) in healthy participants (see Additional file 1 for our PRISMA checklist).

\section{Data extraction and risk of bias assessment}

One researcher performed trial selection and data extraction (LJ); a second researcher (TJ) checked the selection and extraction; a third researcher (PCG) arbitrated. Cochrane's tool (version 2011) was used for risk of bias assessments [25].

\section{Outcome assessment}

We assessed the following primary outcomes: all-cause mortality, deaths from and incidence of HPV-related cancers, incidence of histologically confirmed carcinoma in situ and moderate intraepithelial neoplasia, fatal harms, serious harms and harms of special interest (anaphylaxis, chronic fatigue syndrome [CFS], complex regional pain syndrome [CRPS], Guillain-Barré syndrome [GBS], postural orthostatic tachycardia syndrome [POTS], premature ovarian failure $[\mathrm{POF}]$ and syncope). Histological outcomes were assessed irrespective of which HPV types were involved.

Secondary outcomes included HPV-related external genital lesions and referral procedures, new onset diseases (reported in the included clinical study reports as 'medically significant conditions' and 'new medical history') and general harms (reported as 'solicited', 'unsolicited' and 'systemic adverse events'). We did not consider cytological, serological or virological outcomes or local harms due to their lower clinical importance.

The clinical study reports included over 3000 different types of harms that were classified with MedDRA (Medical Dictionary for Regulatory Activities) preferred terms. The harms were often incompletely and heterogeneously reported (see Table 1). We extracted and assessed all individual harms classified with MedDRA-preferred terms. We performed meta-analyses for the five most commonly occurring fatal and serious harms, the five fatal and serious harms that the HPV vaccines increased the most and the five fatal and serious harms that the HPV vaccines decreased the most. For new onset diseases and general harms, we performed meta-analyses for the three most common, increased and decreased harms for each category ('medically significant conditions' and 'new medical history'; and 'solicited', 'unsolicited' and 'systemic adverse events'). MedDRA-preferred terms and total harms were reported as the number of participants with one or more harms over the total number of participants.

To check for possible harm clustering on an organ system level, we meta-analysed the MedDRA-preferred terms in their respective system organ classes (for example, the MedDRA-preferred terms 'dizziness', 'pain' and 'syncope' were part of and therefore included in the MedDRA system organ class 'nervous system disorders'). Only Merck clinical study reports included aggregate numbers for participants with MedDRA system organ class harms, and only for new onset diseases ('new medical history') and general harms ('systemic adverse events'). For all GlaxoSmithKline clinical study reports and for serious harms for Merck clinical study reports, we pooled MedDRA-preferred terms in their respective system organ classes. A participant could potentially be included more than once in a separate analysis (e.g. if a participant experienced a serious 'headache' and serious 


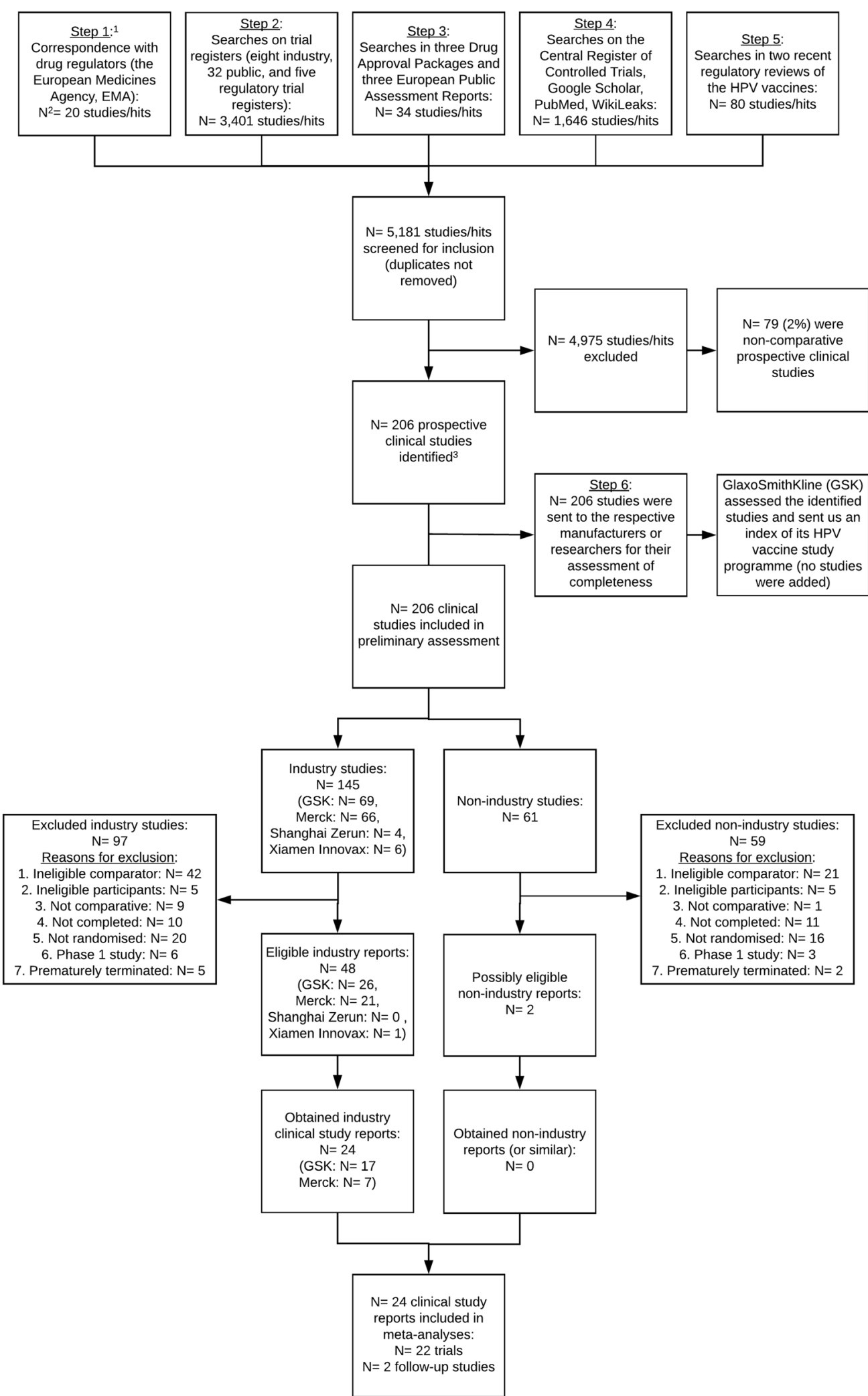

Fig. 1 Benefits and harms of the HPV vaccines: flowchart of the inclusion of clinical study reports. For details on the correspondence and searches conducted in steps 1 to 6, see Jørgensen et al. ([36]: Appendices 1 and 2). Two hundred six studies were identified according to our inclusion and classification criteria, see Jørgensen et al. ([36]: Methods). $N=$ the number of studies/entries evaluated 
Table 1 Benefits and harms of the HPV vaccines: reporting of harms in included HPV vaccine studies

\begin{tabular}{|c|c|c|c|c|c|c|}
\hline \multirow[t]{2}{*}{ Reporting of harms ${ }^{a}$} & \multicolumn{2}{|l|}{ Total } & \multicolumn{2}{|c|}{ GlaxoSmithKline } & \multicolumn{2}{|c|}{ Merck Sharp and Dohme } \\
\hline & $\begin{array}{l}\text { Studies } \\
(N=24)\end{array}$ & $\begin{array}{l}\text { Participants } \\
(N=96,855)^{\mathrm{a}}\end{array}$ & $\begin{array}{l}\text { Studies } \\
(N=17)\end{array}$ & $\begin{array}{l}\text { Participants } \\
(N=66,235)^{a}\end{array}$ & $\begin{array}{l}\text { Studies } \\
(N=7)\end{array}$ & $\begin{array}{l}\text { Participants } \\
(N=30,620)\end{array}$ \\
\hline \multicolumn{7}{|l|}{ Fatal harms } \\
\hline Reported for the whole study period & 23 & $64,679(67 \%)$ & 16 & $34,059(51 \%)$ & 7 & $30,620(100 \%)$ \\
\hline Reported for the whole study period for some ${ }^{b}$ participants & 1 & $32,176(33 \%)$ & 1 & $32,176(49 \%)$ & 0 & $0(0 \%)$ \\
\hline \multicolumn{7}{|l|}{ Serious harms ${ }^{c}$} \\
\hline Reported for the whole study period & 14 & $28,245(30 \%)$ & 14 & $28,245(42 \%)$ & 0 & $0(0 \%)$ \\
\hline No breakdown into MedDRA-preferred terms & $3(21 \%)$ & $2586(9 \%)$ & $3(21 \%)$ & $2586(9 \%)$ & $0(0 \%)$ & $0(0 \%)$ \\
\hline Reported 0 to 14 days post-vaccination & 7 & $30,620(31 \%)$ & 0 & $0(0 \%)$ & 7 & $30,620(100 \%)$ \\
\hline Reported for the 7-month vaccination period & 2 & $5814(6 \%)$ & 2 & $5814(9 \%)$ & 0 & $0(0 \%)$ \\
\hline $\begin{array}{l}\text { Reported for a subset or the serious harms judged } \\
\text { vaccine-related by the trial investigators }{ }^{\mathrm{b}}\end{array}$ & 1 & $32,176(33 \%)$ & 1 & $32,176(49 \%)$ & 0 & $0(0 \%)$ \\
\hline \multicolumn{7}{|l|}{ New onset diseases ${ }^{d}$} \\
\hline $\begin{array}{l}\text { Reported as 'medically significant conditions' for the } \\
\text { whole study period }\end{array}$ & 15 & $65,741(68 \%)$ & 15 & $65,741(99 \%)$ & 0 & $0(0 \%)$ \\
\hline No breakdown into MedDRA-preferred terms & $2(13 \%)$ & $33,216(51 \%)$ & $2(13 \%)$ & $33,216(51 \%)$ & $0(0 \%)$ & $0(0 \%)$ \\
\hline Reported as 'new medical history' for the whole study period & 7 & $30,620(31 \%)$ & 0 & $0(0 \%)$ & 7 & $30,620(100 \%)$ \\
\hline Not reported/included in clinical study report & 2 & $494(1 \%)$ & 2 & $494(1 \%)$ & 0 & $0(0 \%)$ \\
\hline \multicolumn{7}{|l|}{ General harms $^{\mathrm{e}}$} \\
\hline $\begin{array}{l}\text { Reported as 'solicited' and 'unsolicited' general harms } 7 \text { and } \\
30 \text { days post-vaccination }\end{array}$ & 14 & $64,010(66 \%)$ & 14 & $64,010(96 \%)$ & 0 & $0(0 \%)$ \\
\hline Reported for a subset of participants ${ }^{f}$ & $2(14 \%)$ & $7791 / 50,820$ & $2(14 \%)$ & $7791 / 50,820$ & $0(0 \%)$ & $0(0 \%)$ \\
\hline Reported as 'systemic adverse events' 14 days post-vaccination & 7 & $30,620(31 \%)$ & 0 & $0(0 \%)$ & 7 & $30,620(100 \%)$ \\
\hline No breakdown into MedDRA-preferred terms & $3(43 \%)$ & $21,441(70 \%)$ & $0(0 \%)$ & $0(0 \%)$ & $3(43 \%)$ & $21,441(70 \%)$ \\
\hline Not reported/included in clinical study report & 3 & $2225(3 \%)$ & 3 & $2225(4 \%)$ & 0 & $0(0 \%)$ \\
\hline
\end{tabular}

${ }^{\mathrm{a}}$ See Additional file 2 for details on the reporting of harms. Table 1 includes all 24 clinical study reports including the two follow-up studies HPV-023 (followup for trial HPV-001) of 433 participants and HPV-063 (follow-up for trial HPV-032) of 752 participants, i.e. 1185 participants (433 + 752) are included twice for the trials HPV-001 and HPV-032. The total denominator is 95,670 for the 22 included trials and $96,855(95,670+1185)$ for the 24 included clinical study reports ${ }^{b}$ In one trial (HPV-040), 12\% (3703/32,176) of participants were included in a subset population for fatal and serious harms reporting

${ }^{c}(1)$ GlaxoSmithKline defined serious harms as "any untoward medical occurrence that a resulted in death and b was life-threatening, NOTE: The term 'lifethreatening' in the definition of 'serious' refers to an event in which the subject was at risk of death at the time of the event. It did not refer to an event, which hypothetically might have caused death, if it were more severe. c. required hospitalisation or prolongation of existing hospitalisation, NOTE: In general, hospitalisation signified that the subject had been detained (usually involving at least an overnight stay) at the hospital or emergency ward for observation and/or treatment that would not have been appropriate in the physician's office or out-patient setting. Complications that occurred during hospitalisation were AEs [adverse events]. If a complication prolonged hospitalisation or fulfilled any other serious criteria, the event was serious. When in doubt as to whether "hospitalisation" occurred or was necessary, the AE was to be considered serious. Hospitalisation for elective treatment of a pre-existing condition that did not worsen from baseline was not considered an AE. d. resulted in disability/incapacity, NOTE: The term disability means a substantial disruption of a person's ability to conduct normal life functions. This definition was not intended to include experiences of relatively minor medical significance such as uncomplicated headache, nausea, vomiting, diarrhoea, influenza, and accidental trauma (e.g. sprained ankle) which may interfere or prevent everyday life functions but did not constitute a substantial disruption. e. was a congenital anomaly/birth defect in the offspring of a study subject". (2) Merck Sharp and Dohme defined serious harms as "any adverse experience occurring at any dose that: Results in death; or that is life threatening (places the subject/patient, in the view of the investigator, at immediate risk of death from the experience as it occurred. [Note: This does not include an adverse experience that, had it occurred in a more severe form, might have caused death.]); or that results in a persistent or significant disability/incapacity (substantial disruption of one's ability to conduct normal life functions); or that results in or prolongs an existing inpatient hospitalisation (hospitalised is defined as an inpatient admission, regardless of length of stay, even if the hospitalisation is a precautionary measure for continued observation.); or ALSO: Other important medical events that may not result in death, not be life threatening, or not require hospitalisation may be considered a serious adverse experience when, based upon appropriate medical judgement, the event may jeopardise the subject/patient and may require medical or surgical intervention to prevent one of the outcomes listed above"

d(1) GlaxoSmithKline defined 'medically significant conditions' as "Adverse events prompting emergency room or physician visits that are not (1) related to common diseases or (2) routine visits for physical examination or vaccination, or SAEs [serious adverse events] that are not related to common diseases. Serious adverse events related to common diseases were reported but are not classified as medically significant conditions for analysis purposes. Common diseases include: upper respiratory infections, sinusitis, pharyngitis, gastroenteritis, urinary tract infections, cervicovaginal yeast infections, menstrual cycle abnormalities and injury". (2) Merck Sharp and Dohme did not provide a formal definition for 'new medical history' but described 'new medical history' as "all new reported diagnoses" in the clinical study report of trial V501-019

e(1) GlaxoSmithKline defined 'solicited' general adverse events as "Adverse events to be recorded as endpoints in the clinical study [i.e. arthralgia, fatigue, headache, myalgia, pyrexia, rash and urticaria]. The presence/occurrence/intensity of these events is actively solicited from the subject or an observer during a specified post-vaccination follow-up period". (2) GlaxoSmithKline defined 'unsolicited' general adverse event as "Any AE [adverse event] reported in addition to those solicited during the clinical study. Also, any "solicited" symptom with onset outside the specified period of follow-up for solicited symptoms was reported as an unsolicited AE". (3) Merck Sharp and Dohme defined 'systemic adverse event' as "any systemic clinical adverse event that developed on the day of vaccination or during the 14 days after vaccination was recorded on the VRC [vaccination report card] along with the date it started and the last date it was present"

${ }^{f}$ The two trials HPV-008 and HPV-040 only reported general harms for $15 \%(7791 / 50,820)$ of included participants 
'dizziness', the participant would be counted twice in the MedDRA system organ class analysis of serious nervous system disorders); we therefore consider these MedDRA system organ class analyses exploratory.

\section{Post hoc exploratory outcome assessment}

As we did not obtain complete case report forms or individual participant data for any trial, and as the trials' harm assessments had low internal and external validity (see Table 1 and the "Discussion" section), we performed post hoc exploratory outcome analyses where we (1) compared the clinical study report data with pharmacovigilance data; and (2) assessed signs and symptoms of POTS and CRPS (see protocol amendment on PROSPERO [39]).

1) We compared the three largest harm clusters reported from pharmacovigilance up to 1 January 2015 to the World Health Organisation's (WHO) VigiBase ${ }^{\oplus}$ [29] with the clinical study report data (for example, VigiBase's largest HPV vaccine harm cluster-'expected systemic reactions'-consists of the MedDRA-preferred terms headache, nausea, pyrexia, dizziness and vomiting). This was done to assess if the pharmacovigilance data were comparable to the clinical study report data. We used the individual harm cluster terms and found the corresponding MedDRA-preferred terms in the clinical study report data. The data were synthesised or those MedDRApreferred terms included in each harm cluster.

2) POTS and CRPS are rare syndromes that are difficult to identify; as mentioned, about 30 cases of POTS and CRPS were not recognised in the HPV vaccine manufacturers' trials [31, 32], and there were no reports of POTS and CRPS in the clinical study reports (see Table 9 and the "Results" section). To assess whether signs and symptoms consistent with POTS and CRPS were present in the data, we asked a physician (Louise Brinth) with clinical expertise in POTS and CRPS to assess the reported MedDRA-preferred terms as 'definitely', 'probably', 'probably not' or 'definitely not' associated with the syndromes. As an example, the physician judged the MedDRA-preferred terms 'dizziness postural' and 'pain in extremity' to be 'definitely' associated with POTS and CRPS, respectively. The physician was blinded to the allocation groups and outcome data. The data was synthesised for those MedDRA-preferred terms that the physician judged 'definitely' associated with POTS or CRPS. (Note that the synthesis of two or more different MedDRA-preferred term categories may include a participant more than once in an analysis.)

\section{Data synthesis and analysis}

Risk ratios were meta-analysed with the random-effects inverse variance method. As small trials carry more weight with this method, we compared random-effects to a fixed-effect risk ratio for all outcomes. Absolute risk estimates were calculated as the number needed to vaccinate $(\mathrm{NNV})$ or harm $(\mathrm{NNH})$. Review Manager 5 was used for data synthesis and the intention to treat principle to calculate effect estimates. Sensitivity and subgroup analyses were conducted to investigate potential sources of heterogeneity by taking account of age, gender, risk of bias [25] and type of HPV vaccine and comparator.

\section{Results}

\section{Characteristics of included trials}

We identified 50 eligible studies: 43 industry trials, 5 industry follow-up studies and 2 non-industry trials (see Fig. 1). We obtained 24 clinical study reports of 58,412 pages from EMA and GlaxoSmithKline for 22 industry trials and 2 industry follow-up studies (17 Cervarix ${ }^{\mathrm{Ts}}, 5$ Gardasil $^{\mathrm{TM}}, 1$ Gardasil $9^{\mathrm{Tm}}$ and 1 monovalent Merck HPV type 16 vaccine) with a total of 95,670 participants $(79,102$ females and 16,568 males age 8-72) and 393,194 person-years (see Tables 2 and 3 and Additional file 2). The 24 clinical study reports included $79 \%(95,670 /$ $121,441)$ of the total eligible sample of the 50 identified eligible studies. It is possible that for some of these eligible studies clinical study reports were never written but journal articles were published. The mean follow-up time was 49 months (weighted by sample size). About two fifths of the participants in the control groups received the aluminium-based adjuvants that were used in the HPV vaccines $(18,192 / 48,595)$, three fifths received hepatitis vaccines that also contained the aluminium-based adjuvants that were used in the HPV vaccines-except for the hepatitis vaccine Aimmugen ${ }^{\mathrm{Tm}}-(29,500)$, and less than a thousand participants received carrier solution (597) or saline placebo (306).

\section{Characteristics of potentially eligible studies}

For the 26 remaining and potentially eligible studies (23 trials and three follow-up studies) for which no clinical study reports were obtained (or similar reports for the two non-industry trials), numbers of participants were identified for 20 of the 23 industry and 1 of the 2 non-industry trials. The trials included 25,632 and 139 participants, respectively, which were equal to $21 \%$ of the total eligible sample $(25,771 / 121,441)$. These studies were not included in the review or analyses (see Additional file 3).

\section{Risk of bias of included trials}

All 22 trials and the 2 follow-up studies were at low risk of bias for 'sequence generation' and 'allocation concealment', 
Table 2 Benefits and harms of the HPV vaccines: number of pages obtained of clinical study reports from the European Medicines Agency and GlaxoSmithKline

\begin{tabular}{|c|c|c|c|c|c|}
\hline & HPV vaccine manufacturer & Study programme ID & Total pages obtained & European Medicines Agency & GlaxoSmithKline \\
\hline 1 & GlaxoSmithKline & HPV-001 & 5813 & 5813 & 0 \\
\hline 2 & GlaxoSmithKline & HPV-003 & 799 & 0 & 799 \\
\hline 3 & GlaxoSmithKline & HPV-008 & 11,456 & 4263 & 7193 \\
\hline 4 & GlaxoSmithKline & HPV-013 & 8323 & 382 & 7941 \\
\hline 5 & GlaxoSmithKline & HPV-015 & 6290 & 543 & 5747 \\
\hline 6 & GlaxoSmithKline & HPV-023 & 936 & 0 & 936 \\
\hline 7 & GlaxoSmithKline & HPV-029 & 1543 & 0 & 1543 \\
\hline 8 & GlaxoSmithKline & HPV-030 & 1351 & 0 & 1351 \\
\hline 9 & GlaxoSmithKline & HPV-031 & 476 & 0 & 476 \\
\hline 10 & GlaxoSmithKline & HPV-032 & 2912 & 0 & 2912 \\
\hline 11 & GlaxoSmithKline & HPV-033 & 587 & 0 & 587 \\
\hline 12 & GlaxoSmithKline & HPV-035 & 451 & 0 & 451 \\
\hline 13 & GlaxoSmithKline & HPV-038 & 957 & 0 & 957 \\
\hline 14 & GlaxoSmithKline & HPV-040 & 2892 & 128 & 2764 \\
\hline 15 & GlaxoSmithKline & HPV-058 & 1745 & 0 & 1745 \\
\hline 16 & GlaxoSmithKline & HPV-063 & 1474 & 0 & 1474 \\
\hline 17 & GlaxoSmithKline & HPV-069 & 819 & 0 & 819 \\
\hline 18 & Merck Sharp and Dohme & V501-005 & 357 & 357 & 0 \\
\hline 19 & Merck Sharp and Dohme & V501-013 & 1797 & 1797 & 0 \\
\hline 20 & Merck Sharp and Dohme & V501-015 & 713 & 713 & 0 \\
\hline 21 & Merck Sharp and Dohme & V501-018 & 1014 & 1014 & 0 \\
\hline 22 & Merck Sharp and Dohme & V501-019 & 2645 & 2645 & 0 \\
\hline 23 & Merck Sharp and Dohme & V501-020 & 2595 & 2595 & 0 \\
\hline \multirow[t]{2}{*}{24} & Merck Sharp and Dohme & V503-006 & 467 & 467 & 0 \\
\hline & Total pages obtained & & 58,412 & 20,717 & 37,695 \\
\hline
\end{tabular}

and the majority were at low risk of bias for 'blinding of outcome assessors' (19/24) and 'blinding of participants and personnel' (16/24; see Figs. 2 and 3 and Additional file 2). However, due to the following reasons, we judged all studies to be at high risk of bias. Nearly all control participants $(48,289 / 48,595,99 \%)$ received an active comparator such as HPV vaccine aluminium-containing adjuvants or hepatitis vaccines. This distorted-to an unknown extent-the assessment of harms, as the trials tested an HPV vaccine vs. an active part of the same HPV vaccine (see reference [38] for additional clarification). Furthermore, serious harms were incompletely reported for $72 \%$ of the participants $(68,610 / 95,670$; see Table 1 and Additional file 2). All 24 clinical study reports contained redactions-especially of harms-and lacked significant parts such as serious harm narratives and case report forms (except for two reports: HPV-001 and HPV-008, which, however, included less than half of the participants' case report forms) [38]. These situations are not covered by Cochrane's risk of bias tool version 2011. Although not related to participant attrition, we judged the lack of serious harm narratives and case report forms as high risk of 'incomplete outcome data'. In addition, while not related to the availability of study protocols, we judged the redactions of the clinical study reports as high risk of 'selective outcome reporting'. We decided to conduct meta-analyses, since the high risk of bias mainly constituted situations that to our knowledge are not related to empirically verified bias mechanisms.

\section{Benefits}

Seven clinical study reports assessed histological outcomes of which four reported HPV-related cancer outcomes irrespective of involved HPV types. At 4 years follow-up, the HPV vaccines did not decrease HPV-related cancer (7 in the HPV vaccine groups vs. 3 in the comparator groups, risk ratio [RR] 1.68 [95\% confidence interval, $\mathrm{CI}, 0.51$ to 5.49 ], $P=0.39, I^{2}=0 \%$ ) or deaths hereof ( 2 vs. 1 , RR 1.44 [95\% CI 0.23 to 9.12], $P$ 
Table 3 Benefits and harms of the HPV vaccines: characteristics of included participants

\begin{tabular}{|c|c|c|c|c|c|c|c|c|c|}
\hline \multirow{2}{*}{$\begin{array}{l}\text { Characteristics of included } \\
\text { participants }^{\mathrm{a}}\end{array}$} & \multicolumn{2}{|l|}{ Total } & \multicolumn{4}{|l|}{ HPV vaccine } & \multicolumn{3}{|l|}{ Comparator } \\
\hline & $\begin{array}{l}\text { HPV vaccine } \\
(N=47,075)\end{array}$ & $\begin{array}{l}\text { Comparator } \\
(N=48,595)\end{array}$ & $\begin{array}{l}\text { Cervarix } \\
(N=31,316)\end{array}$ & $\begin{array}{l}\text { Gardasil } \\
(N=13,937)\end{array}$ & $\begin{array}{l}\text { Gardasil } 9 \\
(N=618)\end{array}$ & $\begin{array}{l}\text { HPV } 16 \text { vaccine } \\
(N=1204)\end{array}$ & $\begin{array}{l}\text { Placebo } \\
(N=306)\end{array}$ & $\begin{array}{l}\text { Adjuvant }^{\mathrm{b}} \\
(N=18,789)\end{array}$ & $\begin{array}{l}\text { Hepatitis vaccine } \\
(N=29,500)\end{array}$ \\
\hline \multicolumn{10}{|l|}{ Participation } \\
\hline Randomised & 47,075 & 48,595 & 31,316 & 13,937 & 618 & 1204 & 306 & 18,789 & 29,500 \\
\hline $\begin{array}{l}\text { Received one (1) } \\
\text { dose }\end{array}$ & 47,012 (99\%) & 48,556 (99\%) & 31,291 (99\%) & 13,927 (99\%) & 615 (99\%) & 1193 (99\%) & $306(100 \%)$ & 18,750 (99\%) & $29,500(100 \%)$ \\
\hline $\begin{array}{l}\text { Received two (2) } \\
\text { doses }\end{array}$ & 46,105 (98\%) & 47,725 (98\%) & 30,788 (98\%) & 13,564 (97\%) & 604 (98\%) & 1092 (91\%) & 304 (99\%) & 18,304 (97\%) & 29,117 (99\%) \\
\hline $\begin{array}{l}\text { Received three (3) } \\
\text { doses }\end{array}$ & 45,079 (96\%) & $46,726(96 \%)$ & $30,073(96 \%)$ & 13,286 (95\%) & 597 (97\%) & 1019 (85\%) & $300(98 \%)$ & 17,906 (96\%) & 28,520 (97\%) \\
\hline $\begin{array}{l}\text { Completed } \\
\text { vaccination period }\end{array}$ & 44,202 (94\%) & 45,862 (94\%) & 29,331 (94\%) & 13,156 (94\%) & 595 (97\%) & 993 (82\%) & 300 (98\%) & $17,809(95 \%)$ & 27,753 (94\%) \\
\hline $\begin{array}{l}\text { Entered follow-up } \\
\text { period }\end{array}$ & 18,540 (39\%) & $18,059(37 \%)$ & 4090 (14\%) & 13,344 (96\%) & $\begin{array}{l}\text { Not } \\
\text { applicable }\end{array}$ & 1126 (94\%) & $\begin{array}{l}\text { Not } \\
\text { applicable }\end{array}$ & $17,590(94 \%)$ & $469(2 \%)$ \\
\hline $\begin{array}{l}\text { Completed follow-up } \\
\text { period }\end{array}$ & $15,826(34 \%)$ & 14,601 (30\%) & $2929(10 \%)$ & $11,986(86 \%)$ & $\begin{array}{l}\text { Not } \\
\text { applicable }\end{array}$ & 835 (69\%) & $\begin{array}{l}\text { Not } \\
\text { applicable }\end{array}$ & 14,445 (77\%) & $156(1 \%)$ \\
\hline \multicolumn{10}{|l|}{ Gender } \\
\hline Female & 42,036 (89\%) & $37,066(76 \%)$ & $28,876(92 \%)$ & 11,338 (81\%) & $618(100 \%)$ & $1204(100 \%)$ & $306(100 \%)$ & $16,481(88 \%)$ & 20,279 (69\%) \\
\hline \multicolumn{10}{|l|}{ Age } \\
\hline Mean age in years & 20.3 & 20.2 & 21.2 & 21.4 & 19.0 & 20.0 & 19.0 & 22.9 & 20.5 \\
\hline $\begin{array}{l}\text { Age group range in } \\
\text { years }\end{array}$ & $9-72$ & $8-68$ & 9-72 & $9-45$ & $12-26$ & $16-25$ & $12-26$ & $9-68$ & $8-46$ \\
\hline \multicolumn{10}{|l|}{ Race } \\
\hline Asian & 7589 (16\%) & 7295 (15\%) & $6232(20 \%)$ & $1248(9 \%)$ & $40(6 \%)$ & $69(6 \%)$ & $14(5 \%)$ & $2678(14 \%)$ & $4603(16 \%)$ \\
\hline Black & $1426(3 \%)$ & 1492 (3\%) & 467 (2\%) & $862(6 \%)$ & $3(1 \%)$ & $94(8 \%)$ & $3(1 \%)$ & 1108 (6\%) & 381 (1\%) \\
\hline Hispanic & $4492(10 \%)$ & 4378 (9\%) & $1787(6 \%)$ & $2616(19 \%)$ & $0(0 \%)$ & $89(7 \%)$ & $0(0 \%)$ & $3403(18 \%)$ & 975 (3\%) \\
\hline White & $31,743(67 \%)$ & $33,558(69 \%)$ & 22,335 (70\%) & 7998 (56\%) & 483 (78\%) & 918 (76\%) & 231 (75\%) & 9960 (53\%) & 23,367 (79\%) \\
\hline Other & 1625 (3\%) & 1576 (3\%) & 297 (1\%) & 1202 (9\%) & $92(15 \%)$ & $34(3 \%)$ & 58 (19\%) & 1343 (7\%) & 174 (1\%) \\
\hline Unknown & 209 (1\%) & $296(1 \%)$ & 198 (1\%) & $11(1 \%)$ & $0(0 \%)$ & $0(0 \%)$ & $0(0 \%)$ & $297(2 \%)$ & $0(0 \%)$ \\
\hline
\end{tabular}

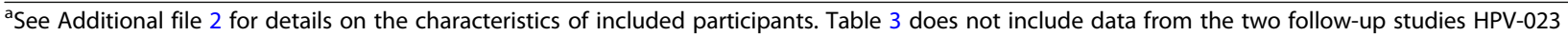
(follow-up for trial HPV-001) of 433 participants and HPV-063 (follow-up for trial HPV-032) of 752 participants

${ }^{b}$ Adjuvant comparators included amorphous aluminium hydroxyphosphate sulphate (AAHS), aluminium hydroxide (Al[OH $]_{3}$ ) and Gardasil's carrier solution (yeast protein, sodium chloride, L-histidine, polysorbate 80 and sodium borate)

${ }^{C}$ Hepatitis vaccines included Aimmugen ${ }^{T M}$ (hepatitis A vaccine), Engerix $-B^{T M}$ (hepatitis B vaccine), Havrix ${ }^{T M}$ (hepatitis A vaccine) and Twinrix Paediatric ${ }^{T M}$ (hepatitis A and B vaccine); see Additional file 2

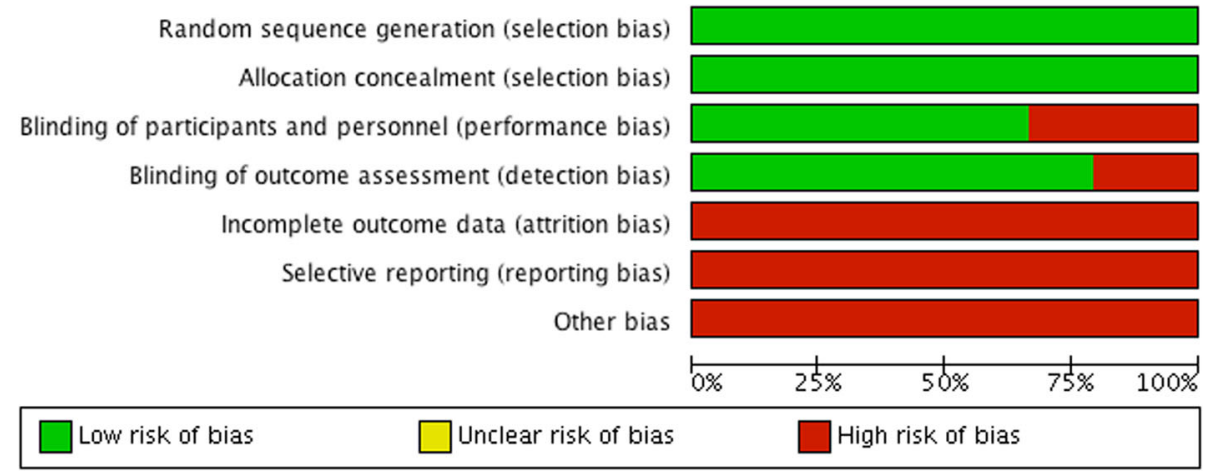

Fig. 2 Benefits and harms of the HPV vaccines: risk of bias graph 


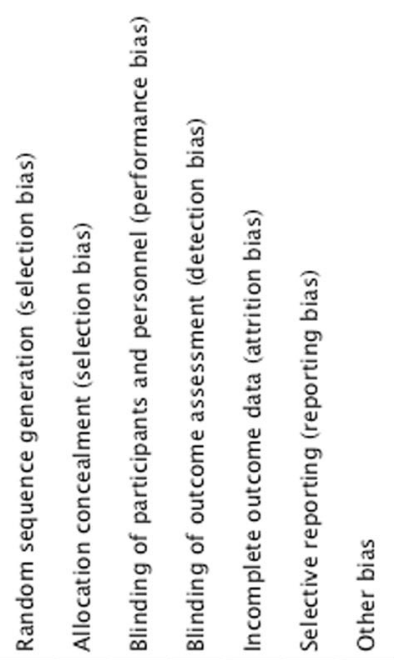

HPV-001: Cenvarix vs. $\mathrm{A}(\mathrm{OH}) 3$ (f, 15-26; 27)

HPV-003: Cenvarix vs. $\mathrm{A}(\mathrm{OH}) 3$ (f, 18-30; 12)

HPV-008: Cenvarix vs. Hawrix (f, 15-25; 48) HPV-013: Cenvarix vs. Hawix (f, 10-14; 12)

HPV-015: Cenvarix vs. $\mathrm{Al}(\mathrm{OH}) 3$ (f, 24-72; 36) HPV-023: Cenvarix vs. Al(OH)3 (f, 21-32; 36) HPV-029: Cenvarix vs. Twinrix (f, 8-15; 12) HPV-030: Cenvarix ws. Engerix (f, 9-15; 12) HPV-031: Cenvarix vs. Al(OH)3 (f, 18-35; 7) HPV-032: Cenvarix vs. Aimmugen (f, 19-25; 7) HPV-033: Cenvarix vs. Hawrix (f, 9-15; 7) HPV-035: Cenvarix vs. $\mathrm{Al}(\mathrm{OH}) 3$ (f, 18-35; 7) HPV-038: Cenvarix vs. $\mathrm{Al}(\mathrm{OH}) 3$ (f, 15-25; 7) HPV-040: Cenvarix ws. Engerix (f \& $\mathrm{m}, 12-16 ; 72$ ) HPV-058: Cenvarix vs. $\mathrm{A}(\mathrm{OH}) 3$ (f, 9-17; 12) HPV-063: Cenvarix vs. Aimmugen (f, 22-29; 12) HPV-069: Cenvarix ws. Engerix (f, 26-46; 12) V501-005: HPV 16 vaccine vs. AAHS (f, 16-25; 48) V501-013: Gardasil ws. AAHS (f, 16-24; 45) V501-015: Gardasil vs. AAHS (f, 15-26; 36) V501-018: Gardasil vs. carrier solu. (f $\& \mathrm{~m}, 9-16 ; 18$ ) V501-019: Gardasil vs. AAHS (f, 21-46; 48) V501-020: Gardasil ws. AAHS (m, 15-27; 36) V503-006: Gardasil 9 vs. saline (f, 12-26;7)

\begin{tabular}{|c|c|c|c|c|c|c|}
\hline & $\oplus$ & $\oplus$ & $\oplus$ & ○ & $\odot$ & \\
\hline & 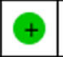 & (๑) & ๑ & - & ○ & C \\
\hline & $\odot$ & $\oplus$ & $\odot$ & $\odot$ & $\odot$ & e \\
\hline & $\odot$ & (?) & 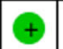 & $\ominus$ & ○ & e \\
\hline+ & $\odot$ & $\hookrightarrow$ & $\hookrightarrow$ & $\odot$ & $\odot$ & e \\
\hline+ & 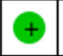 & $\oplus$ & 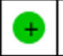 & 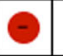 & ○ & e \\
\hline+ & (†) & 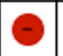 & 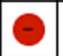 & ○ & ○ & e \\
\hline+ & $\odot$ & ○ & $\theta$ & 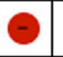 & ○ & ? \\
\hline$\hookrightarrow$ & ๑ & $\oplus$ & 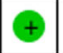 & $\theta$ & $\ominus$ & e \\
\hline+ & 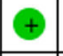 & 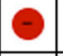 & $\ominus$ & $\theta$ & $\odot$ & $\theta$ \\
\hline$\hookrightarrow$ & 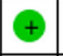 & $\theta$ & $\odot$ & 0 & $\odot$ & $\odot$ \\
\hline+ & 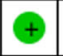 & 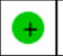 & + & (-) & $\odot$ & e \\
\hline$\hookrightarrow$ & $\odot$ & 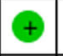 & + & 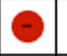 & $\odot$ & e \\
\hline$\uparrow$ & $\odot$ & - & 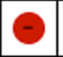 & - & 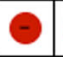 & e \\
\hline+ & ๑ & $\odot$ & 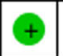 & $\theta$ & $\odot$ & ○ \\
\hline$\hookrightarrow$ & 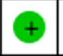 & ( & $\ominus$ & (-) & $\odot$ & - \\
\hline+ & $\odot$ & $\theta$ & $\odot$ & $\theta$ & $\odot$ & - \\
\hline+ & $\odot$ & $\odot$ & $\odot$ & $\theta$ & $\odot$ & 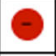 \\
\hline+ & $\odot$ & $\oplus$ & $\odot$ & $\theta$ & $\odot$ & $\theta$ \\
\hline+ & 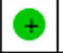 & $\odot$ & 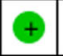 & C & $\odot$ & ○ \\
\hline+ & $\odot$ & 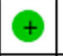 & $\odot$ & $\odot$ & $\odot$ & e \\
\hline+ & 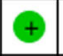 & 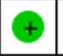 & $\oplus$ & - & $\odot$ & C \\
\hline+ & $\odot$ & $\oplus$ & 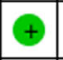 & (-) & 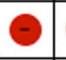 & \\
\hline & ๑ & ๑ & 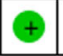 & & & \\
\hline
\end{tabular}

Fig. 3 Benefits and harms of the HPV vaccines: risk of bias summary (each study is noted as "manufacturer ID: type of HPV vaccine vs. type of comparator (included gender, age group; months of follow-up)", e.g. "HPV-001: Cervarix vs. $\mathrm{Al}(\mathrm{OH})_{3}(\mathrm{f}, 15-26 ; 27)^{\prime \prime}$ ) 
Table 4 Benefits and harms of the HPV vaccines: summary of HPV-related outcomes

\begin{tabular}{|c|c|c|c|}
\hline Summary of HPV-related outcomes ${ }^{a}$ & HPV vaccine $(N=47,075)$ & Comparator $(N=48,595)$ & Risk ratio $^{c}[95 \% \mathrm{Cl}]$ \\
\hline \multicolumn{4}{|l|}{ Cancer mortality } \\
\hline Total & 2 & 1 & $1.44[0.23,9.12]$ \\
\hline Cervical & $1(50 \%)$ & $0(0 \%)$ & $2.99[0.12,73.33]$ \\
\hline Oropharyngeal & $1(50 \%)$ & $1(100 \%)$ & $1.00[0.10,9.58]$ \\
\hline \multicolumn{4}{|l|}{ Cancer incidence } \\
\hline Total & 7 & 3 & $1.68[0.51,5.49]$ \\
\hline Anal & $0(0 \%)$ & $0(0 \%)$ & Not applicable \\
\hline Cervical & $3(43 \%)$ & $2(67 \%)$ & $1.41[0.19,10.21]$ \\
\hline Oropharyngeal & $1(14 \%)$ & $1(33 \%)$ & $1.00[0.10,9.58]$ \\
\hline Penile & Not reported & Not reported & Not applicable \\
\hline Vaginal & $1(14 \%)$ & $0(0 \%)$ & $2.99[0.12,73.33]$ \\
\hline Vulvar & $2(29 \%)$ & $0(0 \%)$ & $3.01[0.31,28.89]$ \\
\hline Not HPV-related & 20 & 23 & $0.90[0.49,1.63]$ \\
\hline \multicolumn{4}{|l|}{ Carcinoma in situ incidence } \\
\hline Total & 367 & 490 & $0.73[0.53,1.00]$ \\
\hline Anal (AIN3) & Not reported & Not reported & Not applicable \\
\hline Cervical & $367(100 \%)$ & $490(100 \%)$ & $0.73[0.53,1.00]$ \\
\hline Adenoid type (AIS) & $9(2 \%)$ & $31(6 \%)$ & $0.32[0.15,0.66]$ \\
\hline Squamous type (CIN3) & $358(98 \%)$ & $459(94 \%)$ & $0.85[0.61,1.17]$ \\
\hline Penile (PIN3) & Not reported & Not reported & Not applicable \\
\hline Vaginal (ValN3) & Not reported & Not reported & Not applicable \\
\hline Vulvar (VIN3) & Not reported & Not reported & Not applicable \\
\hline \multicolumn{4}{|l|}{ Moderate intraepithelial neoplasia incidence } \\
\hline Total & 538 & 763 & $0.81[0.59,1.11]$ \\
\hline Anal (AIN2) & $0(0 \%)$ & $0(0 \%)$ & Not applicable \\
\hline Cervical (CIN2) & $538(100 \%)$ & $763(100 \%)$ & $0.81[0.59,1.11]$ \\
\hline Penile (PIN2) & Not reported & Not reported & Not applicable \\
\hline Vaginal (ValN2) & Not reported & Not reported & Not applicable \\
\hline Vulvar (VIN2) & Not reported & Not reported & Not applicable \\
\hline \multicolumn{4}{|l|}{ Carcinoma in situ or worse incidence } \\
\hline Total & 372 & 498 & $0.79[0.59,1.05]$ \\
\hline Anal $\left(\mathrm{AIN3}^{+}\right)$ & Not reported & Not reported & Not applicable \\
\hline Cervical $\left(\mathrm{CIN3}^{+}\right.$, AIS included $)$ & $372(100 \%)$ & $498(100 \%)$ & $0.79[0.59,1.05]$ \\
\hline Penile $\left(\mathrm{PIN3}^{+}\right)$ & Not reported & Not reported & Not applicable \\
\hline Vaginal $\left(\right.$ ValN3 $\left.{ }^{+}\right)$ & Not reported & Not reported & Not applicable \\
\hline Vulvar $\left(\mathrm{VIN}^{+}\right)$ & Not reported & Not reported & Not applicable \\
\hline \multicolumn{4}{|c|}{ Moderate intraepithelial neoplasia or worse incidence } \\
\hline Total & 952 & 1239 & $0.78[0.66,0.91]$ \\
\hline Anal $\left(\right.$ AIN2 $\left.{ }^{+}\right)$ & $0(0 \%)$ & $0(0 \%)$ & Not applicable \\
\hline Cervical $\left(\mathrm{CIN} 2^{+}\right)$ & $892(93 \%)$ & $1144(92 \%)$ & $0.81[0.68,0.97]$ \\
\hline Penile (PIN2 ${ }^{+}$) & $3(1 \%)$ & $3(1 \%)$ & $1.00[0.20,4.95]$ \\
\hline Vaginal $\left(\right.$ ValN2 $\left.{ }^{+}\right)$ & $17(2 \%)$ & $27(2 \%)$ & $0.64[0.32,1.27]$ \\
\hline Vulvar $\left(\mathrm{VIN2}^{+}\right)$ & $18(2 \%)$ & $36(3 \%)$ & $0.49[0.18,1.36]$ \\
\hline Vaginal or vulvar $\left(\mathrm{VIN}^{+}\right.$or ValN2 $\left.2^{+}\right)$ & $22(2 \%)$ & $29(2 \%)$ & $0.76[0.44,1.32]$ \\
\hline
\end{tabular}


Table 4 Benefits and harms of the HPV vaccines: summary of HPV-related outcomes (Continued)

\begin{tabular}{llll}
\hline Summary of HPV-related outcomes ${ }^{\mathrm{a}}$ & HPV vaccine $(\mathrm{N}=47,075)$ & Comparator $(\mathrm{N}=48,595)$ & Risk ratio $^{\mathrm{c}}[95 \% \mathrm{Cl}]$ \\
\hline $\begin{array}{l}\text { External genital lesion (EGL) incidence } \\
\text { Total }\end{array}$ & 289 & 582 & $0.56[0.39,0.82]$ \\
HPV-related referral procedures & & \\
Any & 1941 & 2264 & $0.86[0.81,0.90]$ \\
Biopsy & 2449 & 3021 & $0.74[0.62,0.88]$ \\
Endoscopy & 4354 & 4965 & $0.88[0.85,0.91]$ \\
Treatment (surgical and non-surgical) & 1018 & 1416 & $0.71[0.63,0.80]$ \\
\hline
\end{tabular}

${ }^{a}$ See Additional file 4 sections 1 to 8 for meta-analyses of HPV-related outcomes. It was not feasible to present this summary table for the 16 subgroups (based on age group, gender, type of HPV vaccine and comparator) of the 24 included clinical study reports

bTwo trials (V501-013 and V501-015) reported 'any' procedure, while other trials reported individual outcomes, for example, 'biopsy'

${ }^{\mathrm{c}}$ Risk ratios were calculated with the random-effects inverse variance method

$\left.=0.70, I^{2}=0 \%\right)$; whereas they decreased HPV-related carcinoma in situ (367 vs. 490 , RR 0.73 [95\% CI 0.53 to $1.00]$, number needed to vaccinate $[\mathrm{NNV}] 387, P=0.05$, $\left.I^{2}=67 \%\right)$ and the composite surrogate outcome of HPV-related moderate intraepithelial neoplasia or worse (952 vs. 1239, RR 0.78 [95\% CI 0.66 to 0.91$]$, NNV 190, $\left.P=0.002, I^{2}=53 \%\right)$. The HPV vaccines also decreased HPV-related external genital lesions (289 vs. 582, RR 0.56 [95\% CI 0.39 to 0.82$]$, NNV 47, $P=0.003, I^{2}=83 \%$ ) and HPV-related treatment procedures such as cervical conisations (1018 vs. 1416, RR 0.71 [95\% CI 0.63 to $0.80]$, NNV 75, $P<0.00001, I^{2}=45 \%$ ) (see Table 4 and Additional file 4).

\section{Harms}

\section{Serious harms}

The HPV vaccines did not significantly increase fatal harms ( 45 vs. 38 , RR 1.19 [95\% CI 0.65 to 2.19], $P=$ $\left.0.58, I^{2}=30 \%\right)$ or serious harms (1404 vs. 1357 , RR 1.01 [95\% CI 0.94 to 1.08 ] $P=0.79, I^{2}=0 \%$ ), and no individual fatal or serious harm classified with a MedDRA-preferred term was significantly increased or decreased by the HPV vaccines (see Table 5 and Additional file 4).

\section{New onset diseases}

The HPV vaccines increased new onset back pain (397 vs. 336, RR 1.15 [95\% CI 1.00 to 1.33], NNH 589, $P=$ $\left.0.05, I^{2}=0 \%\right)$ but decreased new onset gynaecological chlamydia infection (1409 vs. 1512, RR 0.93 [95\% CI 0.87 to 1.00$]$, NNV $\left.176, P=0.05, I^{2}=0 \%\right)$ and vaginal infection (369 vs. $420,0.87$ [ $95 \% \mathrm{CI} 0.76$ to 1.00 ], NNV $150, P=0.05, I^{2}=0 \%$ ) (see Table 6 and Additional file 4).

\section{General harms}

The HPV vaccines increased general harms $(13,248$ vs. 12,394, RR 1.07 [95\% CI 1.03 to 1.11], NNH 51, $P$ $\left.=0.0002, I^{2}=77 \%\right)$-especially myalgia (3989 vs. 3047 , RR 1.41 [95\% CI 1.24 to 1.60], NNH 26, $P<0.00001$, $I^{2}=80 \%$ ), fatigue (4933 vs. 4489 , RR 1.13 [95\% CI 1.08 to 1.18$]$, NNH 67, $\left.P<0.00001, I^{2}=22 \%\right)$ and headache (5561 vs. 5246, RR 1.06 [95\% CI 1.02 to 1.11], NNH 83, $P=0.009, I^{2}=40 \%$ ) (see Table 7 and Additional file 4).

\section{Exploratory harm analyses}

The HPV vaccines increased serious nervous system disorders grouped in the MedDRA system organ class (72 vs. 46, RR 1.49 [95\% CI 1.02 to 2.16], number needed to harm [NNH] 1325, $\left.P=0.04, I^{2}=0 \%\right)$ but decreased new onset vascular disorders grouped in the MedDRA system organ class (234 vs. 294, RR 0.80 [95\% CI 0.67 to 0.94], NNV 439, $P=0.009, I^{2}=0 \%$ ) (see Table 8 and Additional file 4).

\section{Harms of special interest}

Cases of anaphylaxis and syncope were evenly distributed. There were no cases of chronic fatigue syndrome (CFS), complex regional pain syndrome (CRPS), Guillain-Barré syndrome (GBS) or postural orthostatic tachycardia syndrome (POTS), but there was one case of premature ovarian failure (POF) in the HPV vaccine group (see Table 9 and Additional file 4).

\section{Post hoc exploratory harm analyses of special interest}

The data from the included clinical study reports that corresponded to the three largest harm clusters reported from pharmacovigilance were associated with general harms, but not serious harms or new onset diseases. The serious harms that were judged 'definitely associated' with POTS or CRPS by the blinded physician were increased by the HPV vaccines, both for POTS (56 vs. 26 , RR 1.92 [95\% CI 1.21 to 3.07], NNH 1073, $P=0.006$, $I^{2}=0 \%$ ) and CRPS (95 vs. 57, RR 1.54 [95\% CI 1.11 to 2.14], NNH 906, $P=0.010, I^{2}=0 \%$ ). The new onset diseases that were judged 'definitely associated' with POTS were also increased by the HPV vaccines (3675 vs. 3352, RR 1.08 [95\% CI 1.01 to 1.15], NNH 144, $P=0.03$, $\left.\mathrm{I}^{2}=29 \%\right)($ see Table 9 and Additional file 4). 
Table 5 Benefits and harms of the HPV vaccines: summary of fatal and serious harms

\begin{tabular}{|c|c|c|c|}
\hline Summary of fatal and serious harms ${ }^{a}$ & HPV vaccine $(N=47,075)$ & Comparator $(N=48,595)$ & Risk ratio $^{\mathrm{e}}[95 \% \mathrm{Cl}]$ \\
\hline \multicolumn{4}{|l|}{ Fatal harms } \\
\hline Participants with fatal harms ${ }^{b}$ & 45 & 38 & $1.19[0.65,2.19]$ \\
\hline Number of MedDRA-classified fatal harms ${ }^{\mathrm{b}}$ & 79 & 51 & Not applicable \\
\hline Number of fatal harms judged HPV vaccine-related & $0(0 \%)$ & $0(0 \%)$ & Not applicable \\
\hline \multicolumn{4}{|c|}{ Most common fatal harms (MedDRA-preferred terms, $n=$ participants) } \\
\hline Cardiorespiratory arrest & 3 & 2 & $0.99[0.13,7.65]$ \\
\hline Completed suicide & 4 & 8 & $0.58[0.15,2.19]$ \\
\hline Gunshot wound & 2 & 3 & $0.74[0.09,5.85]$ \\
\hline Homicide & 2 & 2 & $0.95[0.14,6.50]$ \\
\hline Road traffic accident & 5 & 7 & $0.77[0.24,2.46]$ \\
\hline \multicolumn{4}{|c|}{ Fatal harms most increased by the HPV vaccines (MedDRA-preferred terms, $n=$ participants) } \\
\hline Cardiac arrest & 2 & 0 & $3.00[0.31,28.82]$ \\
\hline Metastases to lung & 2 & 0 & $3.00[0.31,28.82]$ \\
\hline Renal failure acute & 2 & 0 & $3.00[0.31,28.82]$ \\
\hline Systemic lupus erythematosus & 2 & 0 & $3.00[0.31,28.82]$ \\
\hline Traumatic intracranial haemorrhage & 2 & 0 & $3.00[0.31,28.82]$ \\
\hline \multicolumn{4}{|c|}{ Fatal harms most decreased by the HPV vaccines (MedDRA-preferred terms, $n=$ participants) $^{c}$} \\
\hline Completed suicide & 4 & 8 & $0.58[0.15,2.19]$ \\
\hline Gunshot wound & 2 & 3 & $0.74[0.09,5.85]$ \\
\hline Road traffic accident & 5 & 7 & $0.77[0.24,2.46]$ \\
\hline \multicolumn{4}{|l|}{ Serious harms } \\
\hline Participants with serious harms ${ }^{d}$ & 1404 & 1357 & $1.01[0.94,1.08]$ \\
\hline Participants that withdrew due to a serious harm & $54(4 \%)$ & $49(4 \%)$ & $1.08[0.72,1.61]$ \\
\hline Number of MedDRA-classified serious harms ${ }^{d}$ & 1741 & 1628 & Not applicable \\
\hline Number of serious harms judged HPV vaccine-related & $46(3 \%)$ & $44(3 \%)$ & Not applicable \\
\hline \multicolumn{4}{|c|}{ Most common serious harms (MedDRA-preferred terms, $n=$ participants) } \\
\hline Abortion missed & 33 & 41 & $0.81[0.51,1.27]$ \\
\hline Abortion spontaneous & 89 & 78 & $1.14[0.84,1.55]$ \\
\hline Abortion spontaneous complete & 63 & 62 & $1.01[0.71,1.44]$ \\
\hline Abortion spontaneous incomplete & 73 & 54 & $1.35[0.95,1.92]$ \\
\hline Appendicitis & 72 & 82 & $0.85[0.62,1.17]$ \\
\hline \multicolumn{4}{|c|}{ Serious harms most increased by the HPV vaccines (MedDRA-preferred terms, $n=$ participants) } \\
\hline Abortion spontaneous & 89 & 78 & $1.14[0.84,1.55]$ \\
\hline Abortion spontaneous incomplete & 73 & 54 & $1.35[0.95,1.92]$ \\
\hline Pneumonia & 26 & 15 & $1.64[0.87,3.09]$ \\
\hline Pyelonephritis & 31 & 17 & $1.70[0.93,3.10]$ \\
\hline Tonsillitis & 18 & 9 & $1.59[0.72,3.49]$ \\
\hline \multicolumn{4}{|c|}{ Serious harms most decreased by the HPV vaccines (MedDRA-preferred terms, $n=$ participants) } \\
\hline Abortion missed & 33 & 41 & $0.81[0.51,1.27]$ \\
\hline Appendicitis & 72 & 82 & $0.85[0.62,1.17]$ \\
\hline Ligament rupture & 5 & 12 & $0.44[0.15,1.29]$ \\
\hline Ovarian cyst rupture & 6 & 13 & $0.46[0.18,1.21]$ \\
\hline Overdose & 22 & 31 & $0.72[0.42,1.23]$ \\
\hline
\end{tabular}

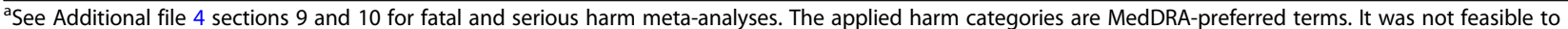
present this summary table for the 16 subgroups (based on age group, gender, type of HPV vaccine and comparator) of the 24 included clinical study reports

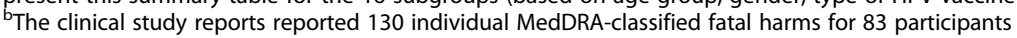

'There were 20 different MedDRA-preferred term categories of fatal harms with the same non-significant difference, i.e. no fatal harm in the HPV vaccine group and one fatal harm in the comparator group

${ }^{\mathrm{d}}$ The clinical study reports reported 3369 individual MedDRA-classified serious harms for 2761 participants, i.e. 1.2 serious harms per participant. Each MedDRAclassified serious harm was reported as the number of participants with a MedDRA-classified serious harm over the total number of participants

e Risk ratios were calculated with the random-effects inverse variance method 
Table 6 Benefits and harms of the HPV vaccines: summary of new onset diseases

\begin{tabular}{|c|c|c|c|c|c|}
\hline Summary of new onset diseases ${ }^{a}$ & $\begin{array}{l}\text { HPV vaccine total } \\
(N=47,075)\end{array}$ & $\begin{array}{l}\text { Comparator total } \\
(N=48,595)\end{array}$ & $\begin{array}{l}\text { Risk ratio }^{f} \text { total } \\
{[95 \% \mathrm{CI}]}\end{array}$ & $\begin{array}{l}\text { Risk ratio }^{f} \text { MSC } \\
{[95 \% \mathrm{CI}]}\end{array}$ & $\begin{array}{l}\text { Risk ratio }^{f} \mathrm{NMH} \\
{[95 \% \mathrm{Cl}]}\end{array}$ \\
\hline \multicolumn{6}{|l|}{ Total } \\
\hline Participants with new onset diseases ${ }^{b}$ & 14,258 & 14,014 & $0.99[0.97,1.02]$ & $0.98[0.90,1.06]$ & $1.00[0.97,1.03]$ \\
\hline Follow-up ${ }^{c}$ & 2296 & 2365 & $0.98[0.94,1.01]$ & Not applicable & $0.98[0.94,1.01]$ \\
\hline Number of MedDRA-classified new onset diseases ${ }^{b}$ & 47,474 & 46,662 & Not applicable & Not applicable & Not applicable \\
\hline Medically significant conditions (MSC) ${ }^{d}$ & $7882(17 \%)$ & $7994(17 \%)$ & Not applicable & Not applicable & Not applicable \\
\hline New medical history $(\mathrm{NMH})^{\mathrm{e}}$ & $39,592(83 \%)$ & $38,668(83 \%)$ & Not applicable & Not applicable & Not applicable \\
\hline \multicolumn{6}{|c|}{ Most common new onset diseases (MedDRA-preferred terms, $n=$ participants) } \\
\hline \multicolumn{6}{|l|}{ MSC } \\
\hline Depression & 443 & 432 & $1.02[0.89,1.16]$ & $1.02[0.85,1.23]$ & $1.01[0.84,1.22]$ \\
\hline Genitourinary tract gonococcal infection & 149 & 162 & $0.92[0.74,1.15]$ & $0.91[0.73,1.14]$ & $1.15[0.37,3.52]$ \\
\hline Gynaecological chlamydia infection & 1409 & 1512 & $0.93[0.87,1.00]$ & $0.95[0.88,1.03]$ & $0.87[0.76,1.00]$ \\
\hline \multicolumn{6}{|l|}{ NMH } \\
\hline Vaginal candidiasis & 1297 & 1359 & $0.95[0.89,1.02]$ & Not applicable & $0.95[0.89,1.02]$ \\
\hline Vaginitis bacterial & 1185 & 1204 & $0.98[0.91,1.06]$ & Not applicable & $0.98[0.91,1.06]$ \\
\hline Urinary tract infection & 1023 & 1086 & $0.93[0.86,1.01]$ & $0.33[0.01,8.19]$ & $0.93[0.86,1.02]$ \\
\hline
\end{tabular}

New onset diseases most increased by the HPV vaccines (MedDRA-preferred terms, $n=$ participants)

MSC

$\begin{array}{llllll}\text { Abdominal pain } & 433 & 374 & 1.21[0.98,1.50] & 1.38[1.00,1.92] & 1.17[0.87,1.57] \\ \text { Back pain } & 397 & 336 & 1.15[1.00,1.33] & 1.40[1.05,1.86] & 1.08[0.91,1.28] \\ \text { Headache } & 771 & 693 & 1.06[0.92,1.22] & 1.29[0.75,2.24] & 1.04[0.93,1.15] \\ \text { MH } & & & & & \\ \text { Amenorrhoea } & 394 & 359 & 1.09[0.87,1.37] & 0.66[0.38,1.15] & 1.17[0.93,1.48] \\ \text { Headache } & 771 & 693 & 1.06[0.92,1.22] & 1.29[0.75,2.24] & 1.04[0.93,1.15] \\ \text { Joint sprain } & 113 & 83 & 1.18[0.80,1.75] & 0.60[0.29,1.22] & 1.45[0.94,2.24]\end{array}$

New onset diseases most decreased by the HPV vaccines (MedDRA-preferred terms, $n=$ participants)

MSC

Cystitis

Gynaecological chlamydia infection

Type 2 diabetes mellitus

$\mathrm{NMH}$

$\begin{array}{lll}\text { Urinary tract infection } & 1023 & 1086 \\ \text { Vaginal candidiasis } & 1297 & 1359 \\ \text { Vaginal infection } & 369 & 420\end{array}$

aSee Additional file 4 section 11 for meta-analyses of new onset diseases. The applied harm categories are MedDRA-preferred terms. New onset diseases consist of 'medically significant conditions' (MSC) and 'new medical history' (NMH). Numbers for 'HPV vaccine' and 'comparator' are the total of MSC and NMH. We divided new onset diseases for MSC and NMH, since the definitions for MSC and NMH differed (see Table 1). It was not feasible to present this summary table for the 16 subgroups (based on age group, gender, type of HPV vaccine and comparator) of the 24 included clinical study reports

${ }^{\mathrm{b}}$ The clinical study reports reported 94,136 individual MedDRA-preferred term classified new onset diseases for 28,272 participants, i.e. 3.3 new onset diseases per participant. New onset diseases were reported as the number of participants over the total number of participants

'Follow-up' represents the trials V501-005, V501-019 and V501-020 that had dichotomized reporting of new medical history (NMH) into the vaccination period (day 0 to month 7) and follow-up period (from month 7 to the last day of follow-up). We included the vaccination periods for these trials in 'participants with new onset diseases' and included the follow-up periods in 'follow-up'

dGlaxoSmithKline defined 'medically significant conditions' as "Adverse events prompting emergency room or physician visits that are not (1) related to common diseases or (2) routine visits for physical examination or vaccination, or SAEs [serious adverse events] that are not related to common diseases. Serious adverse events related to common diseases were reported but are not classified as medically significant conditions for analysis purposes. Common diseases include: upper respiratory infections, sinusitis, pharyngitis, gastroenteritis, urinary tract infections, cervicovaginal yeast infections, menstrual cycle abnormalities and injury" "Merck Sharp and Dohme did not provide a formal definition for 'new medical history' but described 'new medical history' as "all new reported diagnoses" in the clinical study report of trial V501-019

${ }^{f}$ Risk ratios were calculated with the random-effects inverse variance method 
Table 7 Benefits and harms of the HPV vaccines: summary of general harms

\begin{tabular}{|c|c|c|c|c|c|c|}
\hline Summary of general harms ${ }^{\mathrm{a}}$ & $\begin{array}{l}\text { HPV vaccine total } \\
(N=47,075)\end{array}$ & $\begin{array}{l}\text { Comparator total } \\
(N=48,595)\end{array}$ & $\begin{array}{l}\text { Risk ratio }{ }^{f} \text { total } \\
{[95 \% \mathrm{Cl}]}\end{array}$ & $\begin{array}{l}\text { Risk ratiof SGAE } \\
{[95 \% \mathrm{CI}]}\end{array}$ & $\begin{array}{l}\text { Risk ratio }{ }^{f} \text { UGAE } \\
{[95 \% \mathrm{CI}]}\end{array}$ & $\begin{array}{l}\text { Risk ratio }^{f} \text { SYAE } \\
{[95 \% \mathrm{CI}]}\end{array}$ \\
\hline \multicolumn{7}{|l|}{ Total } \\
\hline Participants with general harms ${ }^{b}$ & 13,248 & 12,394 & $1.07[1.03,1.11]$ & $1.11[1.06,1.16]^{9}$ & $1.11[1.06,1.16]^{9}$ & $1.01[0.98,1.03]$ \\
\hline $\begin{array}{l}\text { Number of MedDRA-classified gen- } \\
\text { eral harms } s^{b}\end{array}$ & 37,999 & 31,916 & Not applicable & Not applicable & Not applicable & Not applicable \\
\hline $\begin{array}{l}\text { Solicited general adverse events } \\
(\mathrm{SGAE})^{c}\end{array}$ & $30,408(80 \%)$ & $25,300(79 \%)$ & Not applicable & Not applicable & Not applicable & Not applicable \\
\hline $\begin{array}{l}\text { Unsolicited general adverse events } \\
\left(_{(U G A E)^{d}}\right.\end{array}$ & $3197(8 \%)$ & $3136(10 \%)$ & Not applicable & Not applicable & Not applicable & Not applicable \\
\hline Systemic adverse events (SYAE) ${ }^{\mathrm{e}}$ & $4394(12 \%)$ & $3480(11 \%)$ & Not applicable & Not applicable & Not applicable & Not applicable \\
\hline \multicolumn{7}{|c|}{ Most common general harms (MedDRA-preferred terms, $n=$ participants) } \\
\hline \multicolumn{7}{|l|}{ SGAE and UGAE } \\
\hline Fatigue & 4933 & 4489 & $1.13[1.08,1.18]$ & $1.14[1.09,1.19]$ & $1.00[0.15,6.53]$ & $0.92[0.70,1.20]$ \\
\hline Headache & 5561 & 5246 & $1.06[1.02,1.11]$ & $1.08[1.03,1.14]$ & $1.76[1.26,2.47]$ & $0.98[0.90,1.07]$ \\
\hline Myalgia & 3989 & 3047 & $1.41[1.24,1.60]$ & $1.42[1.24,1.63]$ & $1.15[0.24,5.57]$ & $1.33[0.95,1.85]$ \\
\hline \multicolumn{7}{|l|}{ SYAE } \\
\hline Headache & 5561 & 5246 & $1.06[1.02,1.11]$ & $1.08[1.03,1.14]$ & $1.76[1.26,2.47]$ & $0.98[0.90,1.07]$ \\
\hline Pyrexia & 1599 & 1386 & $1.12[1.02,1.22]$ & $1.15[1.06,1.25]$ & $1.47[0.93,2.34]$ & $1.05[0.80,1.36]$ \\
\hline Nasopharyngitis & 339 & 277 & $1.17[0.91,1.50]$ & Not applicable & $1.40[0.94,2.09]$ & $0.95[0.78,1.16]$ \\
\hline \multicolumn{7}{|c|}{ General harms most increased by the HPV vaccines (MedDRA-preferred terms, $n=$ participants) } \\
\hline \multicolumn{7}{|l|}{ SGAE and UGAE } \\
\hline Fatigue & 4933 & 4489 & $1.13[1.08,1.18]$ & $1.14[1.09,1.19]$ & $1.00[0.15,6.53]$ & $0.92[0.70,1.20]$ \\
\hline Headache & 5561 & 5246 & $1.06[1.02,1.11]$ & $1.08[1.03,1.14]$ & $1.76[1.26,2.47]$ & $0.98[0.90,1.07]$ \\
\hline Myalgia & 3989 & 3047 & $1.41[1.24,1.60]$ & $1.42[1.24,1.63]$ & $1.15[0.24,5.57]$ & $1.33[0.95,1.85]$ \\
\hline \multicolumn{7}{|l|}{ SYAE } \\
\hline Myalgia & 3989 & 3047 & $1.41[1.24,1.60]$ & $1.42[1.24,1.63]$ & $1.15[0.24,5.57]$ & $1.33[0.95,1.85]$ \\
\hline Nausea & 213 & 148 & $1.21[0.89,1.65]$ & Not applicable & $1.32[0.35,4.98]$ & $1.25[0.84,1.86]$ \\
\hline Pyrexia & 1599 & 1386 & $1.12[1.02,1.22]$ & $1.15[1.06,1.25]$ & $1.47[0.93,2.34]$ & $1.05[0.80,1.36]$ \\
\hline \multicolumn{7}{|c|}{ General harms most decreased by the HPV vaccines (MedDRA-preferred terms, $n=$ participants) } \\
\hline \multicolumn{7}{|l|}{ SGAE and UGAE } \\
\hline Influenza & 119 & 120 & $0.91[0.61,1.36]$ & Not applicable & $0.88[0.39,1.97]$ & $0.94[0.56,1.58]$ \\
\hline Cough & 86 & 87 & $0.89[0.65,1.21]$ & Not applicable & $0.83[0.46,1.49]$ & $0.90[0.60,1.37]$ \\
\hline Oropharyngeal pain & 111 & 97 & $1.10[0.80,1.50]$ & Not applicable & $0.91[0.58,1.43]$ & $1.29[0.75,2.22]$ \\
\hline \multicolumn{7}{|l|}{ SYAE } \\
\hline Fungal infection & 4 & 11 & $0.78[0.09,6.43]$ & Not applicable & $3.01[0.31,28.83]$ & $0.18[0.04,0.82]$ \\
\hline Sinus headache & 9 & 15 & $0.49[0.21,1.14]$ & Not applicable & Not applicable & $0.49[0.21,1.14]$ \\
\hline Joint injury & 2 & 5 & $0.47[0.11,2.01]$ & Not applicable & $3.01[0.31,28.83]$ & $0.15[0.03,0.88]$ \\
\hline
\end{tabular}

${ }^{a}$ See Additional file 4 section 12 for meta-analyses of general harms for the 16 subgroups (based on age group, type of HPV vaccine and comparator) of the 24 included clinical study reports. The applied harm categories are MedDRA-preferred terms. The table contains general harms of 'solicited general adverse events' (SGAE), 'unsolicited general adverse events' (UGAE) and 'systemic adverse events' (SYAE). Numbers for 'HPV vaccine' and 'comparator' are the total of SGAE, UGAE and SYAE, but to avoid double counting of participants, UGAE (that accounted for less than $10 \%$ of the general harms) were dismissed from the total risk ratio for studies that reported SGAE and UGAE separately (SGAE and UGAE were not reported as pooled estimates for individual general harms classified with MedDRA-preferred terms; see Additional file 4). It was not feasible to present this summary table for the 16 subgroups (based on age group, type of HPV vaccine and comparator) of the 24 included clinical study reports

${ }^{b}$ The clinical study reports reported 69,915 individual MedDRA-classified general harms for 25,642 participants, i.e. 2.7 general harms per participant. General harms were reported as the number of participants with a MedDRA-classified general harm over the total number of participants

'GlaxoSmithKline defined 'solicited general adverse events' (SGAE) as "Adverse events to be recorded [from day 0 to day 6 after each vaccination] as endpoints [arthralgia, fatigue, headache, myalgia, pyrexia, rash and urticaria] in the clinical study"

'GlaxoSmithKline defined 'unsolicited general adverse events' (UGAE) as "Any AE [adverse event] reported in addition to those solicited during the clinical study. Also, any "solicited" symptom with onset outside the specified period of follow-up for solicited symptoms was reported as an unsolicited AE"

'Merck Sharp and Dohme defined 'systemic adverse events' (SYAE) as "any systemic clinical adverse event that developed on the day of vaccination or during the 14 days after vaccination was recorded on the VRC [vaccination report card]"

${ }^{\mathrm{f}}$ Risk ratios were calculated with the random-effects inverse variance method

'The total numbers of participants with general harms in Cervarix studies were reported as 'solicited [SGAE] and unsolicited [UGAE]', i.e. the risk ratio is similar for SGAE and UGAE 


\section{Subgroup analyses}

Younger HPV vaccinated participants were more protected against moderate HPV-related intraepithelial neoplasia or worse than older participants (age 15 to 29: 784 vs. 1079, RR 0.71 [95\% CI 0.61 to 0.83]; age 21 to 72 : 168 vs. 160 , RR 1.04 [ $95 \%$ CI 0.84 to 1.29 ]; ratio of relative risk [RRR] 1.46 [1.12 to 1.91]) and also experienced fewer fatal harms than older participants (age 15 to 27: 24 vs. 32, RR 0.77 [95\% CI 0.45, 1.33]; age 21 to 72: 21 vs. 6, RR 3.13 [95\% CI 1.29 to 7.61]; RRR 0.25 [95\% CI 0.09 to 0.70$]$ ), but there were no differences for serious nervous system disorders (age 10 to $35: 53$ vs. 35, RR 1.46 [ $95 \%$ CI 0.95 to 2.25 ]; age 21 to $72: 19$ vs. 11 , RR 1.56 [ $95 \%$ CI 0.75 to 3.25]; RRR 0.93 [95\% CI 0.40 to $2.19]$ ), serious harms that were judged 'definitely associated' with of CRPS (age 9 to 35: 76 vs. 48, RR 1.48 [95\% CI 1.03 to 2.12]; age 21 to $72: 19$ vs. 9, RR 2.11 [95\% CI 0.67 to 6.69]; RRR 0.70 [95\% CI 0.21 to 2.34]) or serious harms that were judged 'definitely associated' with POTS (age 12 to $35: 43$ vs. 21, RR 1.86 [95\% CI 1.10, 3.15]; age 21 to $72: 13$ vs. 5, RR 2.22 [95 CI 0.76 to 6.47]; RRR 0.84 [95\% CI 0.25 to 2.76]) (see Additional file 4; note that the subgroup analyses used overlapping age groups due to the different age groups included in the trials). No significant subgroup differences were identified for subgroup analyses based on gender and control treatment.

\section{Random-effects vs. fixed-effect}

We found similar results with the fixed-effect model but with narrower confidence intervals, as the between-trial variance is not included in this model.

\section{Discussion}

Our systematic review of 24 clinical study reports with 95,670 participants showed that the HPV vaccines within 4 years of follow-up decreased HPV-related carcinoma in situ, which have a high likelihood of progressing to cancer [1], and HPV-related treatment procedures, but the vaccines also increased serious nervous system disorders (exploratory analysis) and general harms. Younger participants who are those primarily intended to receive HPV vaccination [1] were more protected against HPV-related neoplasia and had fewer fatal harms.

\section{Strengths}

Our review was based on study programmes, randomised trials reported in clinical study reports, clinically important pre-specified outcomes, intention to treat analyses, absolute risk estimates and a conservative statistical method based on the random-effects model. There was no heterogeneity for serious nervous system disorders or for the post hoc exploratory harm analyses of serious signs and symptoms judged 'definitely associated' with POTS and CRPS by a blinded physician with clinical expertise.

\section{Limitations}

Insufficient trial data access, incomplete reporting, data fragmentation and limited trial follow-up periods were major limitations. It took 3 years to obtain an incomplete subset of the eligible clinical study reports; a process which we have documented in detail elsewhere [38]. Our review is therefore limited by reporting biasthe bias that we aimed to reduce [37]. We did not obtain any periodical safety update reports before our data lock. The inclusion of the remaining participants from the 26 studies with no available clinical study reports included a fifth of the total eligible participants, which could have influenced our review, as some of our results had $P$ values around our cut-off of 0.05 and confidence intervals that were wide.

We performed multiple comparisons: 166 meta-analyses of which $31(19 \%)$ showed statistical significance for the total risk ratio estimate. With our $P$ value cut-off of 0.05 , about eight $(166 * 0.05)$ or a fourth $(8 / 31)$ of the significant results are likely to have occurred by chance. We did not use Bonferroni (or similar) corrections [40], as one of our primary outcomes was serious harms, which were affected by incomplete reporting (see Table 1) and lack of saline placebo controls (see Additional file 2).

The 24 included clinical study reports only included one Gardasil 9 trial (V503-006) that was small and did not investigate histological outcomes. Many countries are currently implementing Gardasil 9 as a two-dose regimen in their vaccination programme instead of Cervarix or Gardasil [1]. Two doses of Gardasil 9 may induce fewer harms than three doses, but Gardasil 9 may induce more harms than Gardasil. For example, in the clinical study report that we obtained of phase 3 multicentre trial V503-001/NCT00543543 (not eligible for our systematic review) of 7106 and 7109 healthy females age 16-26 randomised to receive three doses Gardasil 9 or Gardasil, there were more serious harms (233 vs. 183, RR 1.27 [95\% CI 1.05 to 1.54], NNH 151, $P=0.010$; reported from day 0 to 390) and general harms ('systemic adverse events': 2086 vs. 1929, RR 1.08 [95\% CI 1.03 to 1.14], NNH 75, $P=0.003$; reported $0-14$ days post-vaccination) in the Gardasil 9 group. A 0.5-ml dose of Gardasil 9 contains more virus-like particles $(270 \mu \mathrm{g}$ vs. $100 \mu \mathrm{g})$ and aluminium-containing adjuvant (500 $\mu \mathrm{g}$ vs. $225 \mu \mathrm{g}$ ) compared to a $0.5-\mathrm{ml}$ dose of Gardasil, which could explain the harm differences. Although Gardasil 9 targets five more HPV types than Gardasil, Gardasil 9 did not decrease $\mathrm{CIN}^{+}$more than Gardasil during trial V503-001's 42-month follow-up (325 vs. 326, RR 1.00 [95\% CI 0.86 to 1.16 ], $P=0.97$ ).

A substantial part of our results should be interpreted carefully due to high heterogeneity. We expected the high heterogeneity for several results (e.g. for HPV-related carcinoma in situ), as the included trials comprised 16 
Jørgensen et al. Systematic Reviews

(2020) 9:43

Page 16 of 23

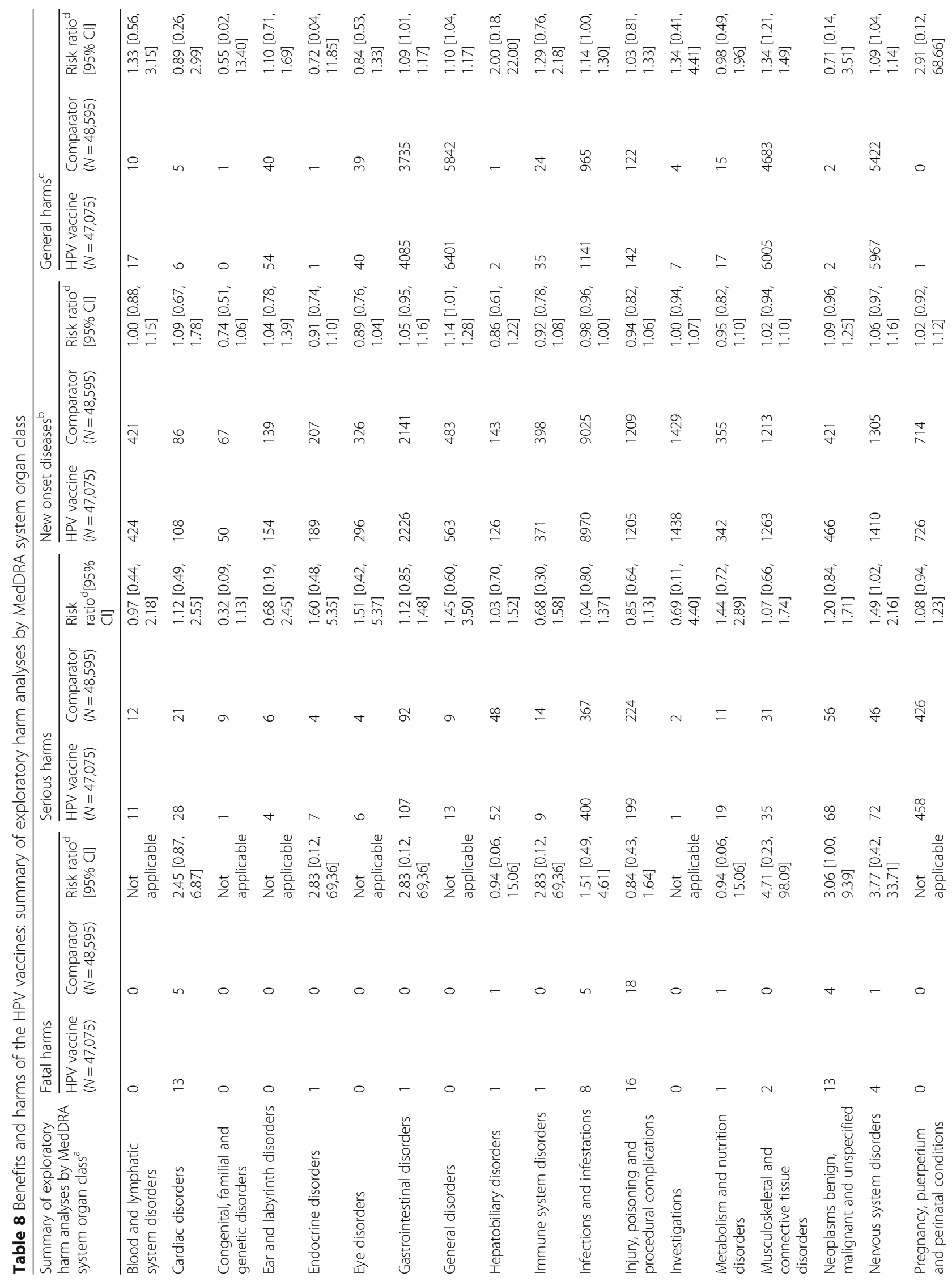




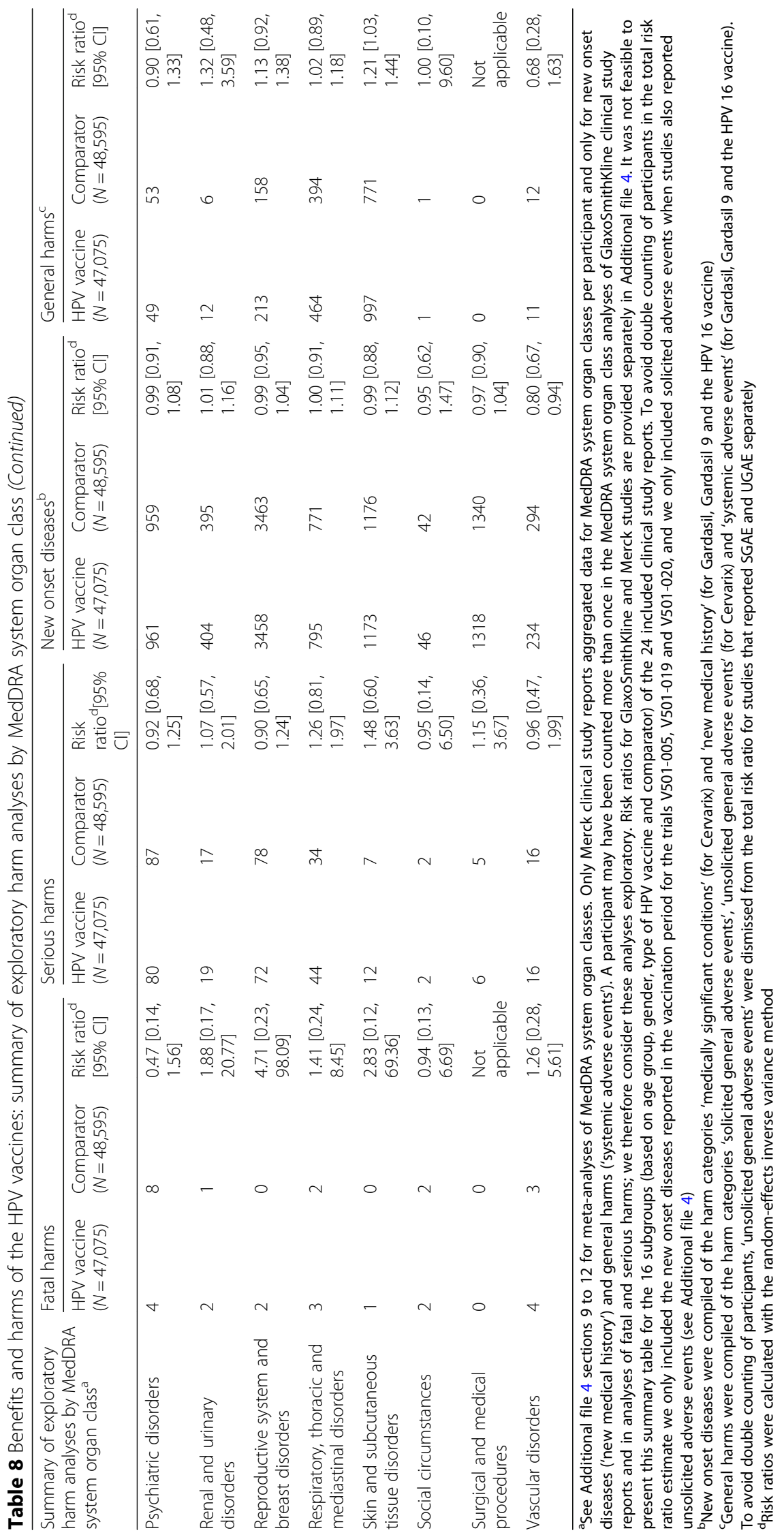


Table 9 Benefits and harms of the HPV vaccines: summary of harms of special interest and post hoc exploratory harm analyses

\begin{tabular}{|c|c|c|c|c|c|c|c|c|c|}
\hline \multirow{2}{*}{$\begin{array}{l}\text { Summary of harms of } \\
\text { special interest and post } \\
\text { hoc exploratory harm } \\
\text { analyses }^{a}\end{array}$} & \multicolumn{3}{|l|}{ Serious harms } & \multicolumn{3}{|c|}{ New onset diseases ${ }^{\mathrm{d}}$} & \multicolumn{3}{|c|}{ General harms ${ }^{e}$} \\
\hline & $\begin{array}{l}\text { HPV vaccine } \\
(N=47,075)\end{array}$ & $\begin{array}{l}\text { Comparator } \\
(N=48,595)\end{array}$ & $\begin{array}{l}\text { Risk } \\
\text { ratiof }^{f}[95 \% \\
\text { Cl] }\end{array}$ & $\begin{array}{l}\text { HPV vaccine } \\
(N=47,075)\end{array}$ & $\begin{array}{l}\text { Comparator } \\
(N=48,595)\end{array}$ & $\begin{array}{l}\text { Risk ratiof } \\
{[95 \% \mathrm{Cl}]}\end{array}$ & $\begin{array}{l}\text { HPV vaccine } \\
(N=47,075)\end{array}$ & $\begin{array}{l}\text { Comparator } \\
(N=48,595)\end{array}$ & $\begin{array}{l}\text { Risk ratio }^{f} \\
{[95 \% \mathrm{Cl}]}\end{array}$ \\
\hline \multicolumn{10}{|c|}{ Harms of special interest (MedDRA-preferred terms, $n=$ participants) } \\
\hline Anaphylaxis & 2 & 4 & $\begin{array}{l}0.59[0.13 \\
2.82]\end{array}$ & 11 & 8 & $\begin{array}{l}1.18[0.48, \\
2.91]\end{array}$ & 0 & 0 & $\begin{array}{l}\text { Not } \\
\text { applicable }\end{array}$ \\
\hline $\begin{array}{l}\text { Chronic fatigue } \\
\text { syndrome (CFS) }\end{array}$ & 0 & 0 & $\begin{array}{l}\text { Not } \\
\text { applicable }\end{array}$ & 0 & 0 & $\begin{array}{l}\text { Not } \\
\text { applicable }\end{array}$ & 0 & 0 & $\begin{array}{l}\text { Not } \\
\text { applicable }\end{array}$ \\
\hline $\begin{array}{l}\text { Chronic regional pain } \\
\text { syndrome (CRPS) }\end{array}$ & 0 & 0 & $\begin{array}{l}\text { Not } \\
\text { applicable }\end{array}$ & 0 & 0 & $\begin{array}{l}\text { Not } \\
\text { applicable }\end{array}$ & 0 & 0 & $\begin{array}{l}\text { Not } \\
\text { applicable }\end{array}$ \\
\hline $\begin{array}{l}\text { Guillain-Barré syndrome } \\
\text { (GBS) }\end{array}$ & 0 & 0 & $\begin{array}{l}\text { Not } \\
\text { applicable }\end{array}$ & 0 & 0 & $\begin{array}{l}\text { Not } \\
\text { applicable }\end{array}$ & 0 & 0 & $\begin{array}{l}\text { Not } \\
\text { applicable }\end{array}$ \\
\hline $\begin{array}{l}\text { Postural orthostatic } \\
\text { tachycardia syndrome } \\
\text { (POTS) }\end{array}$ & 0 & 0 & $\begin{array}{l}\text { Not } \\
\text { applicable }\end{array}$ & 0 & 0 & $\begin{array}{l}\text { Not } \\
\text { applicable }\end{array}$ & 0 & 0 & $\begin{array}{l}\text { Not } \\
\text { applicable }\end{array}$ \\
\hline $\begin{array}{l}\text { Premature ovarian } \\
\text { failure (POF) }\end{array}$ & 0 & 0 & $\begin{array}{l}\text { Not } \\
\text { applicable }\end{array}$ & 1 & 0 & $\begin{array}{l}3.00[0.12, \\
73.48]\end{array}$ & 0 & 0 & $\begin{array}{l}\text { Not } \\
\text { applicable }\end{array}$ \\
\hline Syncope & 4 & 3 & $\begin{array}{l}0.94[0.23 \\
3.81]\end{array}$ & 62 & 60 & $\begin{array}{l}1.03[0.58, \\
1.84]\end{array}$ & 7 & 7 & $\begin{array}{l}0.77[0.25 \\
2.34]\end{array}$ \\
\hline \multicolumn{10}{|c|}{ Post hoc exploratory analyses of VigiBase ${ }^{\oplus}$ harm clusters $^{b}$} \\
\hline $\begin{array}{l}\text { Expected systemic } \\
\text { reactions }\end{array}$ & 25 & 11 & $\begin{array}{l}1.96[0.96 \\
3.98]\end{array}$ & 1465 & 1358 & $\begin{array}{l}1.03[0.93 \\
1.14]\end{array}$ & 10,926 & 9948 & $\begin{array}{l}\text { Not } \\
\text { applicable }\end{array}$ \\
\hline $\begin{array}{l}\text { Allergic/hypersensitivity } \\
\text { reactions }\end{array}$ & 2 & 2 & $\begin{array}{l}0.96[0.14 \\
6.52]\end{array}$ & 284 & 279 & $\begin{array}{l}1.05[0.82, \\
1.35]\end{array}$ & 1912 & 1469 & $\begin{array}{l}1.30[1.18, \\
1.45]\end{array}$ \\
\hline Vasovagal reactions & 9 & 5 & $\begin{array}{l}1.31[0.50 \\
3.46]\end{array}$ & 232 & 212 & $\begin{array}{l}1.06[0.78, \\
1.44]\end{array}$ & 173 & 123 & $\begin{array}{l}1.20[0.93, \\
1.55]\end{array}$ \\
\hline \multicolumn{10}{|c|}{ Post hoc exploratory analyses of CRPS and POTS } \\
\hline $\begin{array}{l}\text { Harms judged as } \\
\text { 'definitely associated' } \\
\text { with CRPS }\end{array}$ & 95 & 57 & $\begin{array}{l}1.54[1.11 \\
2.14]\end{array}$ & 5079 & 4790 & $\begin{array}{l}1.04[0.98 \\
1.10]\end{array}$ & 27,899 & 23,223 & $\begin{array}{l}\text { Not } \\
\text { applicable }\end{array}$ \\
\hline $\begin{array}{l}\text { Harms judged as } \\
\text { 'definitely associated' } \\
\text { with POTS }\end{array}$ & 56 & 26 & $\begin{array}{l}1.92[1.21 \\
3.07]\end{array}$ & 3675 & 3352 & $\begin{array}{l}1.08[1.01 \\
1.15]\end{array}$ & 18,207 & 16,288 & $\begin{array}{l}\text { Not } \\
\text { applicable }\end{array}$ \\
\hline
\end{tabular}

\footnotetext{
${ }^{a}$ See Additional file 4 sections 13 and 14 for meta-analyses of the harms of special interest and post hoc exploratory harm analyses. There was no applicable fatal harm of special interest. It was not feasible to present this summary table for the 16 subgroups (based on age group, gender, type of HPV vaccine and comparator) of the 24 included clinical study reports. As we did not obtain complete case report forms or individual participant data, we could not assign harms to individual participants

${ }^{\mathrm{b}}$ As the included studies' harm assessments were at risk of low internal and external validity (see Table 1 and the "Discussion" section), we compared the three largest harm clusters reported from pharmacovigilance up to 1 January 2015 to the World Health Organisation's (WHO) VigiBase with the clinical study report data. We did this to see if the pharmacovigilance data were similar to the study data. VigiBase's largest HPV vaccine harm cluster (expected systemic reactions) consists of 'headache, nausea, pyrexia, dizziness and vomiting'. VigiBase's second largest HPV vaccine harm cluster (allergic/hypersensitivity reactions) consists of 'pruritis, urticaria, rash and erythema'. VigiBase's third largest HPV vaccine harm cluster (vasovagal reactions) consists of 'syncope, dizziness, loss of consciousness, pallor and seizure'. As we synthesised individual MedDRA-preferred term classified harms, our post hoc exploratory analyses of VigiBase harm clusters may therefore include a participant more than once in each separate analysis

'We asked a physician with clinical expertise in POTS and CRPS to assess the reported MedDRA terms as 'definitely', 'probably', 'probably not' or 'definitely not' associated with the syndromes. The physician was blinded to the allocation groups and outcome data. The data was synthesised for those MedDRA-preferred terms that the physician judged 'definitely' associated with POTS or CRPS. As we synthesised individual MedDRA-preferred terms, our post hoc exploratory analyses of CRPS and POTS may include a participant more than once in each separate analysis

'New onset diseases were compiled of the harm categories 'medically significant conditions' (for Cervarix) and 'new medical history' (for the HPV 16 vaccine, Gardasil and Gardasil 9)

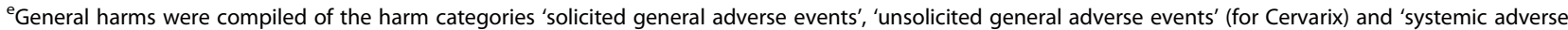
events' (for Gardasil, Gardasil 9 and the HPV 16 vaccine)

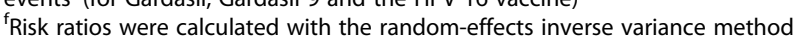

${ }^{9}$ Some numerators exceeded the denominators making the result nonsensical. Therefore, we did not perform meta-analyses
} 
different subgroups-based on the type of HPV vaccine, comparator, age and gender. All meta-analyses were divided according to the 16 subgroups to provide heterogeneity measures (see Additional file 4), but the nationality of the participants and regional practices of HPV-related screening and treatment procedures may also have contributed to the heterogeneity.

\section{Limitations of benefit assessment}

Only $10 \mathrm{HPV}$-related cancers occurred in the follow-up periods. Extended follow-up was not possible for $75 \%$ of the comparator participants $(36,344 / 48,595)$, as they were offered HPV vaccination at trial completion.

We only included benefit results of intention to treat analyses, which also included participants that were enrolled after they had been infected with HPV. The HPV vaccines have no documented effect on HPV-related neoplasia caused by previous infections [1]. Our benefit results may therefore be skewed toward the null compared to real-life settings where mainly 12 -year-old adolescents-that are expected to not be previously HPV-infected-are HPV vaccinated. Getting vaccinated before sexual debut is likely to improve the HPV vaccines' benefits, but no included trial investigated histological outcomes for participants that were vaccinated under the age of 15 .

Three trials-HPV-008, V501-013 and V501-015 that contained $38 \%(36,266 / 95,670)$ of the analysed participants-were stopped early when HPV type 16/18-related cervical intraepithelial neoplasia or worse $\left(\mathrm{CIN}^{+}\right)$was significantly reduced for their HPV vaccine per-protocol populations. On average, trials stopped early for benefits exaggerate effects by $29 \%$ compared to completed trials of the same intervention [41]. When the three trials were excluded from our $\mathrm{CIN}^{+}$meta-analysis, $\mathrm{CIN}^{+}$ was not significantly decreased ( 184 vs. 200 , RR 0.85 [95\% CI 0.54 to 1.33$], \quad P=0.47, \quad I^{2}=77 \%$; see Additional file 4).

One clinical study report (HPV-015) only reported $\mathrm{CIN2}^{+}$, although there were three cases of HPV-related cancers in the HPV vaccine group and one in the comparator group (see Additional file 4). These cancers were listed as serious harms and were not mentioned elsewhere in the clinical study report. For transparency, it would have been more appropriate to report each histological outcome (cancer, carcinoma in situ, moderate intraepithelial neoplasia, etc.) than only a composite surrogate outcome such as CIN2 $2^{+}$.

No trial tested the HPV vaccines' protection against cervical cancer without using cervical screening. This may reduce external validity, as some studies show that HPV-vaccinated women may tend to avoid cervical screening [42]; although other studies have not shown a clear tendency [43]. The trial personnel often performed cervical screening together with colposcopy every 6 months, and the included participants were often women aged 15-26. In clinical practice, cervical screening is usually performed every 3 to 5 years and recommended after age 25 [44], as most $\mathrm{CIN}^{+}$lesions in women under age 30 regress spontaneously, which may justify active surveillance rather than immediate intervention [45].

No trial used mandatory biopsies, which may reduce internal validity. For example, the precursor lesion of cervical adenocarcinoma is difficult to detect on colposcopy, but easier to recognise on a biopsy [46]. The incidence of cervical adenocarcinoma is increasing and may more often be HPV negative compared to cervical squamous carcinoma [46], but only 5\% (40/857) of the reported cervical carcinoma in situ cases in the included studies were adenocarcinoma in situ (see Table 4).

We did not pre-specify genital warts as an outcome, but the HPV vaccines reduced external genital lesions and there is strong evidence that the HPV vaccinesespecially Gardasil and Gardasil 9 that target the HPV types 6 and 11-decrease the incidence of genital warts [47].

\section{Limitations of harm assessment}

Only Merck clinical study reports reported aggregate numbers for participants with MedDRA system organ classified harms, and only for new onset diseases and general harms. The synthesis of MedDRA system organ classes for all GlaxoSmithKline clinical study reports and for serious harms for Merck clinical study reports may therefore include a participant more than once. As a result, we consider these analyses exploratory.

Serious harms were incompletely reported for $72 \%$ of the participants $(68,610 / 95,670$; see Table 1 and Additional file 2). There were 2.8 times more serious harms reported in the clinical study reports that reported serious harms for the whole trial period (1838/27,493 vs. 923/38,356). As an example, trial HPV-008 of Cervarix that had reported all serious harms during its 48 months follow-up reported 10 times more participants with serious harms compared to V501-015 of Gardasil that only reported serious harms 14 days post-vaccination (1664/ $18,644$ vs. $102 / 12,167)$. In the cluster-randomised trial, HPV-040, $88 \%(28,473$ of 32,176$)$ of the participants were not included for serious harms reporting (see Table 1 and Additional file 2).

The use of active comparators may have underestimated harms related to the HPV vaccines [38]. The aluminium-containing comparators were used, as they provided a similar appearance to that of the HPV vaccines, which enhanced blinding and decreased the risk of performance and detection bias. A single trialV503-006, of Gardasil 9-used a saline placebo in 306 
participants who had previously been vaccinated with Gardasil. It is unlikely that those who had experienced harms following previous Gardasil vaccination would have participated in the Gardasil 9 trial, so the trial's harm results are not reliable. The trial's blinding procedure was adequate to ensure low risk of performance and detection bias and could have been used in other trials (see Additional file 2).

Although the manufacturers consider the aluminiumcontaining comparators to be safe, $52 \%$ of the participants $(49,301 / 95,670)$ were only included in the trials if they had never received the aluminium-containing comparators before. GlaxoSmithKline state that their aluminium-containing comparator induces myalgia ("higher incidences of myalgia might namely be attributable to the higher content of aluminium in the HPV vaccine $[450$ micrograms $\mathrm{Al}(\mathrm{OH})_{3}$ ] than the content of aluminium in the HAV [hepatitis A] vaccine [225 micrograms $\left.\mathrm{Al}(\mathrm{OH})_{3}\right]$ " [48]), which we found was increased by the HPV vaccines (see Table 7).

The clinical study reports, their informed consent forms and corresponding journal publications (for example, V501-013 [49] and V501-015 [50]) often used the term placebo (which is a substance with no active effect) to describe the active aluminium-based comparators.

Two thirds of the participants $(63,468 / 95,670)$ were only included in the trials if they had no history of immunological or nervous system disorders (see Additional file 2). Such disorders are not listed as warnings or contraindications on the package inserts of the approved HPV vaccines [8-10]. The degree of harms might therefore be higher in clinical practice than in the trials. The HPV vaccines did not increase the three largest HPV vaccine-related VigiBase ${ }^{\circ}$ harms clusters for serious harms and new onset diseases (see the "Methods" section, Table 9 and Additional file 4), which may reflect the differences between real-life and the trials' settings and entry criteria.

The exploratory analyses of MedDRA system organ classes may have included a participant more than once. For serious nervous system disorders, this is unlikely, as there were only 118 participants with such disorders (reported as individual MedDRA-preferred terms) for 61,331 participants (see Additional file 4). We note, however, that the serious nervous system disorders consisted of very heterogenous harms, for example, 'anoxic encephalopathy', 'moyamoya disease' and 'vertebral artery dissection'.

The serious harm analyses of MedDRA-preferred terms associated with POTS and CRPS may also have included a participant more than once, although this is unlikely as there only were 82 participants with a POTS sign/symptom for 60,058 participants and 152 participants with a CRPS sign/symptom for 60,915 participants. The selection of MedDRA-preferred terms associated with POTS and CRPS was subjective, not verified by other assessors and included some signs/ symptoms that do not align well with the diagnostic criteria of POTS or CRPS [51, 52], for example, 'constipation', 'vision blurred' and 'vomiting'. Other blinded assessors would possibly assign MedDRA-preferred terms differently, as there were over 3000 different included MedDRA-preferred terms. The post hoc exploratory POTS and CRPS analyses were based on randomised trial data where serious harms were underreported and likely underestimated, but since no complete serious harm narratives or complete case report forms were available, the analyses could not take symptom duration, symptom clustering or the diagnostic criteria into account. Therefore, the analyses do not prove that the HPV vaccines cause POTS and CRPS, but they do provide a signal, which makes it important to carry out independent analyses of POTS and CRPS based on the complete data set with individual participant data.

\section{Similar studies}

In May 2018, a Cochrane review of the HPV vaccines that included 26 trials with 73,428 female participants concluded that the HPV vaccines decrease precursors to cervical cancer and do not increase serious or general harms [3]. The Cochrane review had similar inclusion criteria to our review, but it was mainly based on journal publications and only included phase II and III trials. In comparison, we identified 50 possibly eligible studies for which we obtained clinical study reports for 22 trials and two follow-up studies and included 30\% more participants $(95,670)$ than the Cochrane review. We found that the HPV vaccines decrease precursors to HPV-related cancer and treatment procedures but increase serious nervous system disorders (exploratory analyses) and general harms. Another recent review on males [53] and most large epidemiological studies have found no serious harms associated with the HPV vaccines [16-20].

\section{Conclusion}

At 4 years follow-up, the HPV vaccines decreased HPV-related precursors to cervical cancer and treatment procedures but increased serious nervous system disorders (exploratory analysis) and general harms. As the included trials were primarily designed to assess benefits and not adequately designed to assess harms, the extent to which the benefits outweigh the harms is unclear. Limited access to clinical study reports and trial data with case report forms prevented a thorough assessment. An independent assessment of the complete individual participant data is needed. If granted access to the 
complete data set with individual participant data, we will update this systematic review. A large industry-independent multicentre trial of two doses of Gardasil 9 vs. saline placebo would likely be informative in identifying a more accurate benefit-harm balance, but we recognise that such a trial will be considered unethical in most settings.

\section{Additional files}

Additional file 1: Benefits and harms of the HPV vaccines-PRISMA 2009 checklist. (DOCX 31 kb)

Additional file 2: Benefits and harms of the HPV vaccines-characteristics of included studies. (DOCX $121 \mathrm{~kb}$ )

Additional file 3: Benefits and harms of the HPV vaccines-list of excluded studies. (DOCX 63 kb)

Additional file 4: Benefits and harms of the HPV vaccines-metaanalyses. (PDF $26103 \mathrm{~kb}$ )

\begin{abstract}
Abbreviations
AIN: Anal intraepithelial neoplasia; CFS: Chronic fatique syndrome; CIN: Cervica intraepithelial neoplasia; CRPS: Chronic regional pain syndrome; EGL: External genital lesion; EMA: European Medicines Agency; FDA: Food and Drug Administration; GBS: Guillain-Barré syndrome; GSK: GlaxoSmithKline; HPV: Human papillomavirus; ICH: International Council for Harmonisation of Technical Requirements for Pharmaceuticals for Human Use; MedDRA: Medical Dictionary for Regulatory Activities; Merck: Merck and Co., Inc. or Merck Sharp and Dohme outside the USA and Canada; MSC: Medically significant condition; NMH: New medical history; NNH: Number needed to harm; NNV: Number needed to vaccinate; PICO: Patient, intervention, comparator and outcome; PIN: Penile intraepithelial neoplasia; POF: Premature ovarian failure; POTS: Postural orthostatic tachycardia syndrome; PRISMA: Preferred Reporting Items for Systematic Reviews and Meta-Analyses; PROSPERO: International Prospective Register of Systematic Reviews; SGAE: Solicited general adverse event; SYAE: Systemic adverse event; UGAE: Unsolicited general adverse event; ValN: Vaginal intraepithelial neoplasia; VIN: Vulvar intraepithelial neoplasia; WHO: World Health Organisation
\end{abstract}

\section{Acknowledgements}

We would like to thank EMA and Louise Brinth for their assistance and Jeppe Bennekou Schroll and Karsten Juhl Jørgensen for helpful comments.

\section{Funding}

This study was funded by the Nordic Cochrane Centre, which is funded by the Danish government.

\section{Availability of data and materials}

The datasets generated and analysed during the current systematic review are available from the first author $(L J)$ upon request.

\begin{abstract}
Authors' contributions
$\sqcup J$ wrote the first draft. $L J$ and TJ contributed to the conception of the review, the design of the review, the collection and assembly of data, the analysis and interpretation of the data, the drafting of the article, the critical revision of the article for important intellectual content and the final approval of the article. PCG contributed to the conception of the review, the critical revision of the article for important intellectual content and the final approval of the article. All authors had full access to all the data in the study and take responsibility for the integrity of the data and the accuracy of the data analysis.
\end{abstract}

Ethics approval and consent to participate

Not applicable.

\section{Consent for publication}

Not applicable.

\section{Competing interests}

All authors have completed the ICMJE uniform disclosure form. $\amalg J$ declares no support from any organisation for the submitted work, no financial relationships with any organisations that might have an interest in the submitted work, no other relationships or activities that could appear to have influenced the submitted work. PCG spoke by video link about the HPV vaccines at the IFICA (International Federation for Injured Children and Adults, www.ificaeurope.org) conference in 2018 but received no fee or reimbursement for this. PCG and TJ were co-signatories of a complaint to the European Ombudsman on maladministration in relation to the EMA investigation of possible harms from HPV vaccines. PCG does not regard this as a competing interest. TJ was a co-recipient of a UK National Institute for Health Research grant (HTA-10/80/01 update and amalgamation of two Cochrane Reviews: neuraminidase inhibitors for preventing and treating influenza in healthy adults and children-https://www.journalslibrary.nihr.ac. uk/programmes/hta/108001\#/). TJ is also in receipt of a Cochrane Methods Innovations Fund grant to develop guidance on the use of regulatory data in Cochrane reviews. TJ is occasionally interviewed by market research companies about phase I or II pharmaceutical products. In 2011-2014, TJ acted as an expert witness in a litigation case related to the antiviral oseltamivir, in two litigation cases on potential vaccine-related damage and in a labour case on influenza vaccines in healthcare workers in Canada. He has acted as a consultant for Roche (1997-1999), GSK (2001-2002), Sanofi-Synthelabo (2003) and IMS Health (2013). In 2014-2016, TJ was a member of three advisory boards for Boehringer Ingelheim. TJ was a member of an independent data monitoring committee for a Sanofi Pasteur clinical trial on an influenza vaccine.

\section{Publisher's Note}

Springer Nature remains neutral with regard to jurisdictional claims in published maps and institutional affiliations.

\section{Author details}

${ }^{1}$ Nordic Cochrane Centre, Rigshospitalet 7811, Tagensvej 22, 2200 Copenhagen, Denmark. ²Department of Clinical Medicine, Rigshospitalet, Blegdamsvej 9, 2100 København, Denmark. ${ }^{3}$ Institute for Scientific Freedom, Copenhagen, Denmark.

Received: 20 June 2018 Accepted: 6 March 2019

Published online: 28 February 2020

\section{References}

1. World Health Organization. Human papillomavirus vaccines: WHO position paper. Wkly Epidemiol Rec. 2017;92(19):241-68.

2. European Medicines Agency. Review under Article 20 of Regulation (EC) No 726/2004 Human papillomavirus (HPV) vaccines. 2015. Available from: http://www.ema.europa.eu/docs/en_GB/document_ library/Referrals_document/HPV_vaccines_20/Opinion_provided_by_ Committee_for_Medicinal_Products_for_Human_Use/WC500197129. pdf.

3. Arbyn M, Xu L, Simoens C, Martin-Hirsch PPL. Prophylactic vaccination against human papillomaviruses to prevent cervical cancer and its precursors. Cochrane Database Syst Rev. 2018;5:CD009069. https://doi.org/ 10.1002/14651858.CD009069.pub3.

4. Kjaer SK, Nygård M, Dillner J, Brooke Marshall J, Radley D, Li M, et al. A 12year follow-up on the long-term effectiveness of the quadrivalent human papillomavirus vaccine in 4 Nordic countries. Clin Infect Dis. 2018;66(3):33945. https://doi.org/10.1093/cid/cix797.

5. Guo F, Cofie LE, Berenson AB. Cervical Cancer incidence in young U.S. females after human papillomavirus vaccine introduction. Am J Prev Med. 2018;55(2):197-204. https://doi.org/10.1016/j.amepre.2018.03.013.

6. Dehlendorff C, Sparén P, Baldur-Felskov B, Herweijer E, ArnheimDahlström L, Ploner A, et al. Effectiveness of varying number of doses and timing between doses of quadrivalent HPV vaccine against severe cervical lesions. Vaccine. 2018;36(43):6373-8. https://doi.org/10.1016/j. vaccine.2018.09.011

7. Hall MT, Simms KT, Lew J-B, Smith MA, Brotherton JM, Saville M, et al. The projected timeframe until cervical cancer elimination in Australia: a modelling study. Lancet Public Health. 2018. https://doi.org/10.1016/S24682667(18)30183-X. 
8. Research C for BE and. Approved Products - Cervarix Available from: https:// www.fda.gov/BiologicsBloodVaccines/Vaccines/ApprovedProducts/ ucm186957.htm

9. Research C for BE and. Approved Products - Gardasil. Available from: https:// www.fda.gov/BiologicsBloodVaccines/Vaccines/ApprovedProducts/ UCM094042

10. Research C for BE and. Approved Products-Gardasil 9. Available from: https://www.fda.gov/BiologicsBloodVaccines/Vaccines/ApprovedProducts/ ucm426445.htm

11. Pagliusi SR, Teresa Aguado M. Efficacy and other milestones for human papillomavirus vaccine introduction. Vaccine. 2004;23(5):569-78.

12. IARC HPV Working Group. Primary end-points for prophylactic HPV vaccine trials. Lyon: International Agency for Research on Cancer; 2014.

13. de Sanjose S, Quint WG, Alemany L, Geraets DT, Klaustermeier JE, Lloveras B, et al. Human papillomavirus genotype attribution in invasive cervical cancer: a retrospective cross-sectional worldwide study. Lancet Oncol. 2010;11(11): 1048-56. https://doi.org/10.1016/S1470-2045(10)70230-8.

14. Rodríguez-Carunchio L, Soveral I, Steenbergen RDM, Torné A, Martinez S, Fusté $P$, et al. HPV-negative carcinoma of the uterine cervix: a distinct type of cervical cancer with poor prognosis. BJOG. 2015;122(1):119-27. https:// doi.org/10.1111/1471-0528.13071

15. Interim Clinical Study Report for Study 580299/008 (HPV-008). Available from: https://www.gsk-clinicalstudyregister.com/files2/gsk-580299-008clinical-study-report-redact.pdf.

16. Feiring B, Laake I, Bakken IJ, Greve-Isdahl M, Wyller VB, Håberg SE, et al. HPV vaccination and risk of chronic fatigue syndrome/myalgic encephalomyelitis: a nationwide register-based study from Norway. Vaccine. 2017;35(33):4203-12. https://doi.org/10.1016/j.vaccine.2017.06.031.

17. Miranda S, Chaignot C, Collin C, Dray-Spira R, Weill A, Zureik M. Human papillomavirus vaccination and risk of autoimmune diseases: a large cohort study of over 2 million young girls in France. Vaccine. 2017;35(36):4761-8. https://doi.org/10.1016/j.vaccine.2017.06.030.

18. Scheller NM, Pasternak B, Mølgaard-Nielsen D, Svanström H, Hviid A. Quadrivalent HPV vaccination and the risk of adverse pregnancy outcomes. N Engl J Med. 2017;376(13):1223-33. https://doi.org/10.1056/ NEJMoa1612296.

19. Phillips A, Patel C, Pillsbury A, Brotherton J, Macartney K. Safety of human papillomavirus vaccines: an updated review. Drug Saf. 2018;41(4):329-46. https://doi.org/10.1007/s40264-017-0625-z.

20. Liu EY, Smith LM, Ellis AK, Whitaker H, Law B, Kwong JC, et al. Quadrivalent human papillomavirus vaccination in girls and the risk of autoimmune disorders: the Ontario grade $8 \mathrm{HPV}$ vaccine cohort study. CMAJ. 2018;190(21):E648-55. https://doi.org/10.1503/cmaj. 170871.

21. Golder S, Loke YK, Wright K, Norman G. Reporting of adverse events in published and unpublished studies of health care interventions: a systematic review. PLoS Med. 2016;13(9):e1002127. https://doi.org/10.1371/ journal.pmed.1002127.

22. Schroll JB, Penninga El, Gøtzsche PC. Assessment of adverse events in protocols, clinical study reports, and published papers of trials of orlistat: a document analysis. PLoS Med. 2016;13(8):e1002101. https://doi.org/10.1371/ journal.pmed.1002101.

23. Sharma T, Guski LS, Freund N, Gøtzsche PC. Suicidality and aggression during antidepressant treatment: systematic review and meta-analyses based on clinical study reports. BMJ. 2016;27(352):i65. https://doi.org/10. 1136/bmj.i65.

24. Maund E, Tendal B, Hróbjartsson A, Jørgensen KJ, Lundh A, Schroll J, et al. Benefits and harms in clinical trials of duloxetine for treatment of major depressive disorder: comparison of clinical study reports, trial registries, and publications. BMJ. 2014;348:g3510. https://doi.org/10.1136/bmj.g3510.

25. Cochrane Handbook for Systematic Reviews of Interventions. Available from: http://training.cochrane.org/handbook

26. Blitshteyn S, Brook J. Postural tachycardia syndrome (POTS) with anti-NMDA receptor antibodies after human papillomavirus vaccination. Immunol Res. 2017;65(1):282-4. https://doi.org/10.1007/s12026-016-8855-1.

27. Brinth LS, Pors K, Theibel AC, Mehlsen J. Orthostatic intolerance and postural tachycardia syndrome as suspected adverse effects of vaccination against human papilloma virus. Vaccine. 2015;33(22):2602-5. https://doi.org/ 10.1016/j.vaccine.2015.03.098.

28. Kinoshita T, Abe R-T, Hineno A, Tsunekawa K, Nakane S, Ikeda S-I. Peripheral sympathetic nerve dysfunction in adolescent Japanese girls following immunization with the human papillomavirus vaccine. Intern Med. 2015;54(15):1955. https://doi.org/10.2169/internalmedicine.54.4644

29. Chandler RE, Juhlin K, Fransson J, Caster O, Edwards IR, Norén GN. Current safety concerns with human papillomavirus vaccine: a cluster analysis of reports in VigiBase ${ }^{\oplus}$. Drug Saf. 2017;40(1):81-90. https://doi.org/10.1007/ s40264-016-0456-3.

30. Jefferson T, Jørgensen L. Human papillomavirus vaccines, complex regional pain syndrome, postural orthostatic tachycardia syndrome, and autonomic dysfunction - a review of the regulatory evidence from the European Medicines Agency. Indian J Med Ethics. 2017;2(1):30-7. https://doi.org/10. 20529/IJME.2017.006.

31. Weber $C$, Andersen $\mathrm{S}$. The company behind the HPV vaccine underestimated the extent of serious side effects [firma bag HPV-vaccinen underdrev omfanget af alvorlige bivirkninger]. 2015. Available from: https:// www.b.dk/content/item/105701.

32. The Committee for Medicinal Products for Human Use (CHMP) at the European Medicines Agency's (EMA) committee responsible for human medicines. Rapporteurs' Day 150 Joint Response Assessment Report of Gardasil 9. 2014. EMEA/h/c/3852.

33. Palmieri B, Poddighe D, Vadalà M, Laurino C, Carnovale C, Clementi E. Severe somatoform and dysautonomic syndromes after HPV vaccination: case series and review of literature. Immunol Res. 2017;65(1):106-16. https:// doi.org/10.1007/s12026-016-8820-z.

34. Ojha RP, Jackson BE, Tota JE, Offutt-Powell TN, Singh KP, Bae S. Guillain-Barre syndrome following quadrivalent human papillomavirus vaccination among vaccine-eligible individuals in the United States. Hum Vaccin Immunother. 2014;10(1):232-7. https://doi.org/10.4161/hv. 26292.

35. Little DT, Ward HRG. Premature ovarian failure 3 years after menarche in a 16-year-old girl following human papillomavirus vaccination. BMJ Case Rep. 2012, 2012;30. https://doi.org/10.1136/bcr-2012-006879.

36. Jørgensen L, Gøtzsche PC, Jefferson T. Index of the human papillomavirus (HPV) vaccine industry clinical study programmes and non-industry funded studies: a necessary basis to address reporting bias in a systematic review. Syst Rev. 2018;7(1):8. https://doi.org/10.1186/ s13643-018-0675-z.

37. Jørgensen L, Gøtzsche PC, Jefferson T. Benefits and harms of the human papillomavirus vaccines: systematic review of industry and non-industry study reports. York: PROSPERO; 2017. Available from: https://www.crd.york.ac.uk/ PROSPEROFILES/56093_PROTOCOL_20170030.pdf

38. Jørgensen L, Doshi P, Gøtzsche PC, Jefferson T. Challenges of independent assessment of potential harms of HPV vaccine. BMJ. 2018;362:k3694. https:// doi.org/10.1136/bmj.k3694.

39. Jørgensen L, Gøtzsche PC, Jefferson T. Protocol amendment no. 1 and 2: benefits and harms of the human papillomavirus vaccines: systematic review of industry and non-industry study reports. York: PROSPERO; 2017. Available from: https://www.crd.york.ac.uk/PROSPEROFILES/56093_ PROTOCOL_20171116.pdf

40. Polanin JR, Pigott TD. The use of meta-analytic statistical significance testing Res Synth Methods. 2015;6(1):63-73. https://doi.org/10.1002/jrsm.1124.

41. Bassler D, Briel M, Montori VM, Lane M, Glasziou P, Zhou Q, et al. Stopping randomized trials early for benefit and estimation of treatment effects: systematic review and meta-regression analysis. JAMA. 2010;303(12):1180-7. https://doi.org/10.1001/jama.2010.310.

42. Budd AC, Brotherton JML, Gertig DM, Chau T, Drennan KT, Saville M. Cervical screening rates for women vaccinated against human papillomavirus. Med J Aust. 2014;201(5):279-82.

43. Paynter CA, Van Treeck BJ, Verdenius I, Lau AWY, Dhawan T, Lash KA, et al. Adherence to cervical cancer screening varies by human papillomavirus vaccination status in a high-risk population. Prev Med Rep. 2015;2:711-6. https://doi.org/10.1016/j.pmedr.2015.07.011.

44. Dickinson JA, Ogilvie G, Niekerk DV, Popadiuk C. Evidence that supports policies to delay cervical screening until after age 25 years. CMAJ. 2017; 189(10):E380-1. https://doi.org/10.1503/cmaj.160636.

45. Tainio K, Athanasiou A, KAO T, Aaltonen R, Hernándes CJ, et al. Clinical course of untreated cervical intraepithelial neoplasia grade 2 under active surveillance: systematic review and meta-analysis. BMJ. 2018;360:k499. https://doi.org/10.1136/bmj.k499.

46. Serrano B, Brotons M, Bosch FX, Bruni L. Epidemiology and burden of HPVrelated disease. Best Pract Res Clin Obstet Gynaecol. 2018;47:14-26. https:// doi.org/10.1016/j.bpobgyn.2017.08.006. 
47. Harper DM, DeMars LR. HPV vaccines - a review of the first decade. Gynecol Oncol. 2017;146(1):196-204. https://doi.org/10.1016/j.ygyno.2017. 04.004 .

48. GSK Study Register - Study 104951. Available from: https://www.gskclinicalstudyregister.com/study/104951?search=study\&search_terms= 104951\#CSr

49. Garland SM, Hernandez-Avila M, Wheeler CM, Perez G, Harper DM, Leodolter $S$, et al. Quadrivalent vaccine against human papillomavirus to prevent anogenital diseases. N Engl J Med. 2007;356(19):1915-27. https://doi.org/10. 1056/NEJMoa061741.

50. FUTURE II Study Group. Quadrivalent vaccine against human papillomavirus to prevent high-grade cervical lesions. N Engl J Med. 2007;356(19):1915-27. https://doi.org/10.1056/NEJMoa061741.

51. Grubb BP. Postural tachycardia syndrome. Circulation. 2008;117(21):2814-7. https://doi.org/10.1161/CIRCULATIONAHA.107.761643.

52. Harden RN, Bruehl S, Perez RSGM, Birklein F, Marinus J, Maihofner C, et al. Validation of proposed diagnostic criteria (the "Budapest criteria") for complex regional pain syndrome. Pain. 2010;150(2):268-74. https://doi.org/ 10.1016/j.pain.2010.04.030.

53. Harder T, Wichmann O, Klug SJ, van der Sande MAB, Wiese-Posselt M. Efficacy, effectiveness and safety of vaccination against human papillomavirus in males: a systematic review. BMC Med. 2018;16(1):110 https://doi.org/10.1186/s12916-018-1098-3.

Ready to submit your research? Choose BMC and benefit from:

- fast, convenient online submission

- thorough peer review by experienced researchers in your field

- rapid publication on acceptance

- support for research data, including large and complex data types

- gold Open Access which fosters wider collaboration and increased citations

- maximum visibility for your research: over $100 \mathrm{M}$ website views per year

At $\mathrm{BMC}$, research is always in progress.

Learn more biomedcentral.com/submissions 